\title{
On fixed points of a generalized multidimensional affine recursion
}

\author{
Mariusz Mirek
}

Received: 23 October 2011 / Revised: 26 May 2012 / Published online: 6 July 2012

(C) The Author(s) 2012. This article is published with open access at Springerlink.com

\begin{abstract}
Let $G$ be a multiplicative subsemigroup of the general linear group $\mathrm{Gl}\left(\mathbb{R}^{d}\right)$ which consists of matrices with positive entries such that every column and every row contains a strictly positive element. Given a $G$-valued random matrix $A$, we consider the following generalized multidimensional affine equation

$$
R \stackrel{\mathcal{D}}{=} \sum_{i=1}^{N} A_{i} R_{i}+B,
$$

where $N \geq 2$ is a fixed natural number, $A_{1}, \ldots, A_{N}$ are independent copies of $A, B \in$ $\mathbb{R}^{d}$ is a random vector with positive entries, and $R_{1}, \ldots, R_{N}$ are independent copies of $R \in \mathbb{R}^{d}$, which have also positive entries. Moreover, all of them are mutually independent and $\stackrel{\mathcal{D}}{=}$ stands for the equality in distribution. We will show with the aid of spectral theory developed by Guivarc'h and Le Page (Simplicité de spectres de Lyapounov et propriété d'isolation spectrale pour une famille d'opérateurs de transfert sur l'espace projectif. Random Walks and Geometry, Walter de Gruyter GmbH \& Co. KG, Berlin, 2004; On matricial renewal theorems and tails of stationary measures for affine stochastic recursions, Preprint, 2011) and Kesten's renewal theorem (Kesten in Ann Probab 2:355-386, 1974), that under appropriate conditions, there exists $\chi>0$ such that $\mathbb{P}(\{\langle R, u\rangle>t\}) \asymp t^{-\chi}$, as $t \rightarrow \infty$, for every unit vector $u \in \mathbb{S}^{d-1}$ with positive entries.
\end{abstract}

Keywords Heavy tailed random variables · Renewal theory $\cdot$ Stationary measures · Markov chains $\cdot$ Spectral theory

This research project was partially supported by MNiSW Grant N N201 392337.

M. Mirek $(\bowtie)$

Institute of Mathematics, University of Wroclaw, 50-384 Wroclaw, Poland

e-mail: mirek@math.uni.wroc.pl 


\section{Mathematics Subject Classification $\quad 60 \mathrm{H} 25 \cdot 60 \mathrm{~K} 05 \cdot 60 \mathrm{~J} 80$}

\section{Introduction and statement of the results}

We consider the Euclidean space $\mathbb{R}^{d}$ endowed with the scalar product $\langle x, y\rangle=$ $\sum_{i=1}^{d} x_{i} y_{i}$, the norm $|x|=\sqrt{\langle x, x\rangle}$, and its Borel $\sigma$-field $\mathcal{B}$ or $\left(\mathbb{R}^{d}\right)$. We say that $\mathbb{R}^{d} \ni x=\left(x_{1}, \ldots, x_{d}\right) \geq 0$ is positive (resp. $\mathbb{R}^{d} \ni x=\left(x_{1}, \ldots, x_{d}\right)>0$ is strictly positive) when $x_{n} \geq 0$, (resp. $x_{n}>0$ ) for every $1 \leq n \leq d$. By $\mathbb{R}_{+}^{d}$ we denote the set of all positive vectors and we define the set $\mathbb{S}^{+}=\mathbb{R}_{+}^{d} \cap \mathbb{S}^{d-1}$ of all positive vectors on the unit sphere $\mathbb{S}^{d-1}=\left\{x \in \mathbb{R}^{d}:|x|=1\right\}$ with the distance being the restriction of the Euclidean norm to $\mathbb{S}^{+}$. Given $x \in \mathbb{R}^{d}$ we denote its projection on $\mathbb{S}^{d-1}$ by $\bar{x}=\frac{x}{|x|}$.

Let $\mathrm{Gl}\left(\mathbb{R}^{d}\right)$ be the group of $d \times d$ invertible matrices on $\mathbb{R}^{d}$ with the operator norm $\|\cdot\|$ associated with the Euclidean norm $|\cdot|$ on $\mathbb{R}^{d}$, i.e. $\|a\|=\sup _{x \in \mathbb{S} d-1}|a x|$ for every $a=(a(i, j))_{1 \leq i, j \leq d} \in \mathrm{Gl}\left(\mathbb{R}^{d}\right)$.

Suppose that $G$ is a multiplicative subsemigroup of $\operatorname{Gl}\left(\mathbb{R}^{d}\right)$ which consists of matrices with positive entries such that every column and every row contains a strictly positive element. By $G^{\circ}$ we denote the multiplicative subsemigroup of $G$ composed of matrices with strictly positive entries. It is easy to see that $G$ provides a projective action on $\mathbb{S}^{+}$which is given by

$$
G \times \mathbb{S}^{+} \ni(a, x) \mapsto a \cdot x=\frac{a x}{|a x|} \in \mathbb{S}^{+} .
$$

Let $A$ be a $G$-valued random matrix distributed according to a probability measure $\mu$ on $G$, and $B$ be a random vector independent of $A$, taking its values in $\mathbb{R}_{+}^{d}$.

Let $A_{1}, \ldots, A_{N}$ and $B_{0}$ be independent random variables, where $N \geq 2$ is a fixed natural number, $A_{1}, \ldots, A_{N}$ are independent copies of $A$, and $B_{0}$ is an independent copy of $B$.

The aim of this paper is to find a random vector $R \in \mathbb{R}_{+}^{d}$, independent of $A$ and $B$, which solves (in law $\stackrel{\mathcal{D}}{=}$ ) a generalized multidimensional affine equation, i.e.

$$
R \stackrel{\mathcal{D}}{=} \sum_{i=1}^{N} A_{i} R_{i}+B_{0},
$$

where $R_{1}, \ldots, R_{N}$ are independent copies of $R \in \mathbb{R}_{+}^{d}$ and independent of $A, A_{1}, \ldots$, $A_{N}, B, B_{0}$, (see Theorem 1.7 stated below).

Furthermore, we would like to find possibly mild conditions, which allows us to establish an asymptotic tail formula for $R$. More precisely, we are interested in the existence $\chi>0$, such that

$$
\mathbb{P}(\{\langle R, u\rangle>t\}) \asymp t^{-\chi}, \quad \text { as } t \rightarrow \infty,
$$

for every $u \in \mathbb{S}^{+}$(see Theorem 1.9 stated below).

The one dimensional version of Eq. (1.1) has been considered recently by Jelenković and Olvera-Cravioto [13-15] in the context of Google's PageRank algorithm. The 
authors solved Eq. (1.1) and justified formula (1.2) using the renewal theorem. It is worth emphasizing that the one dimensional version of Eq. (1.1) with $B=0$, was studied by Liu in a series of articles (see for instance [18] and the references given there).

We are also motivated by the recent results of Buraczewski et al. [3], where the authors considered the multidimensional version of Eq. (1.1) with $B=0$, and established formula (1.2) with the help of Kesten's renewal theorem [17] and the spectral method developed by Guivarc'h and Le Page [6,7]. Their approach sheds some new light on multidimensional problems and fits perfectly to our situation.

In order to avoid repetitions in the sequel, and shorten article we have decided to state all necessary definitions and notations in the introduction, and formulate our main results as general as it is possible.

Let $M^{1}(G)$ denotes the set of all probability measures on $G$ endowed with the weak topology. We denote by $\operatorname{supp} \mu$ the support of the measure $\mu \in M^{1}(G)$. If $E \subseteq G$, let $[E]$ be the subsemigroup of $G$ generated by the set $E$. For $n \in \mathbb{N}$ let $S_{n}=A_{n} \cdots A_{1} \in G$, where $A_{1}, A_{2}, \ldots \in G$ is a sequence of independent copies of $G$-valued random matrix $A$ distributed according to $\mu$.

A subsemigroup $[\operatorname{supp} \mu]$ of $G$ is called contractive if $[\operatorname{supp} \mu] \cap G^{\circ} \neq \emptyset$. In other words,

$$
\mathbb{P}\left(\bigcup_{n \in \mathbb{N}}\left\{S_{n} \in G^{\circ}\right\}\right)>0 .
$$

The condition (1.3) was considered by Hennion [10], Hennion and Hervé [11] in the context of limit theorems for the products of positive random matrices.

An element $a \in \mathrm{Gl}\left(\mathbb{R}^{d}\right)$ is proximal if there exists a unique eigenvalue $\lambda_{a}$ (the dominant eigenvalue) of $a$, such that $r(a)=\lim _{n \rightarrow \infty}\left\|a^{n}\right\|^{1 / n}=\left|\lambda_{a}\right|$.

According to the Perron-Frobenius theorem [12] every $a \in G^{\circ}$ is proximal. Moreover, for every $a \in G^{\circ}$ and its adjoint $a^{*} \in G^{\circ}$ it is possible to choose $v_{a}, w_{a} \in \mathbb{R}_{+}^{d}$ such that $v_{a}>0, w_{a}>0$ and

$$
a v_{a}=\lambda_{a} v_{a}, \quad a^{*} w_{a}=\lambda_{a} w_{a}, \quad\left\langle v_{a}, w_{a}\right\rangle=1, \quad\left|w_{a}\right|=1 .
$$

The eigenvector $v_{a}$ determined by these relations will be called the dominant eigenvector of $a \in G^{\circ}$. This means that we can write $\mathbb{R}^{d}=\mathbb{R} \cdot v_{a} \oplus v_{a}^{\perp}$, and the spectral radius of $a$ restricted to $v_{a}^{\perp}=\left\{x \in \mathbb{R}^{d}:\left\langle x, v_{a}\right\rangle=0\right\}$ is strictly less than $\left|\lambda_{a}\right|$. Furthermore, by the preceding relations we have

$$
\lim _{n \rightarrow \infty} \frac{a^{n}}{r(a)^{n}}=v_{a} \otimes w_{a}
$$

where $v_{a} \otimes w_{a}$ is the matrix projector on $\mathbb{R} \cdot v_{a}$. Since $v_{a} \otimes w_{a} x=\left\langle x, w_{a}\right\rangle v_{a}$ for every $x \in \mathbb{R}^{d}$, (1.4) immediately yields

$$
\lim _{n \rightarrow \infty} a^{n} \cdot x=\frac{v_{a} \otimes w_{a} x}{\left|v_{a} \otimes w_{a} x\right|}=\frac{v_{a}}{\left|v_{a}\right|}=\bar{v}_{a} \in \mathbb{S}^{+}, \quad \text { for every } x \in \mathbb{R}_{+}^{d} .
$$


A subsemigroup $\Gamma \subseteq \mathrm{Gl}\left(\mathbb{R}^{d}\right)$ is strongly irreducible if there does not exist a finite number $(k \in \mathbb{N})$ of proper linear subspaces $V_{1}, \ldots, V_{k}$ of $\mathbb{R}^{d}$ such that

$$
\Gamma\left(\bigcup_{i=1}^{k} V_{i}\right) \subseteq \bigcup_{i=1}^{k} V_{i}
$$

If $E \subseteq \mathrm{Gl}\left(\mathbb{R}^{d}\right)$ we denote by $E^{\text {prox }}$ the set of all proximal elements of $E$. A subsemigroup $\Gamma \subseteq \mathrm{Gl}\left(\mathbb{R}^{d}\right)$ is said to satisfy condition $(i-p)$ if $\Gamma$ is strongly irreducible and $\Gamma^{\text {prox }} \neq \emptyset$. This condition was widely investigated by Guivarc'h and Le Page [6,7], see also $[3,8,9]$ and the references given there.

A subsemigroup $[\operatorname{supp} \mu] \subseteq G$, where $\mu \in M^{1}(G)$, is said to satisfy condition $(\mathcal{C})$ if $[\operatorname{supp} \mu]$ is contractive and strongly irreducible. Clearly, condition $(\mathcal{C})$ implies condition $(i-p)$ with $\Gamma=[\operatorname{supp} \mu]$.

For $s \geq 0$ we write

$$
\kappa(s)=\kappa_{\mu}(s)=\lim _{n \rightarrow \infty}\left(\int_{G}\|a\|^{s} \mu^{* n}(d a)\right)^{\frac{1}{n}},
$$

where $\mu^{* n}$ is the $n$th convolution power of $\mu \in M^{1}(G)$. The limit above exists and it is equal to $\inf _{n \in \mathbb{N}}\left(\int_{G}\|a\|^{s} \mu^{* n}(d a)\right)^{\frac{1}{n}}$, because $u_{n}(s)=\int_{G}\|a\|^{s} \mu^{* n}(d a)$ is submultiplicative, i.e. $u_{m+n}(s) \leq u_{m}(s) u_{n}(s)$ for every $m, n \in \mathbb{N}$. Moreover,

$$
I_{\mu}=\left\{s \in[0, \infty): \kappa_{\mu}(s)<\infty\right\}=\left\{s \in[0, \infty): \int_{G}\|a\|^{s} \mu(d a)<\infty\right\} .
$$

Let $s_{\infty}=\sup \left\{s \geq 0: \kappa_{\mu}(s)<\infty\right\} \in \mathbb{R}_{+} \cup\{\infty\}$, then by the Hölder inequality $I_{\mu}=\left[0, s_{\infty}\right)$ or $I_{\mu}=\left[0, s_{\infty}\right]$.

Our "existence" result is the following

Theorem 1.7 Assume that $A$ is a $G$-valued random matrix distributed according to a probability measure $\mu$ on $G$, and $B$ is a random vector independent of $A$, taking its values in $\mathbb{R}_{+}^{d}$, such that $\mathbb{P}(\{B>0\})>0$. Let $A_{1}, \ldots, A_{N}$ and $B_{0}$ be independent random variables as in (1.1), where $N \geq 2$ is a fixed natural number, $A_{1}, \ldots, A_{N}$ are independent copies of $A$, and $B_{0}$ is an independent copy of $B$. Suppose further that $[$ supp $\mu] \subseteq G$ satisfies condition $(\mathcal{C})$ and there exist $s_{1} \in(0,1 / 2]$, and $s_{2}>s_{1}$ such that $\mathbb{E}\left(\|A\|^{s_{1}}\right) \leq \frac{1}{N}, \mathbb{E}\left(\|A\|^{s_{2}}\right) \leq \frac{1}{N}$, and $\mathbb{E}\left(|B|^{s_{2}}\right)<\infty$. Then there exists a unique vector $R \in \mathbb{R}_{+}^{d}$ and its independent copies $R_{1}, \ldots, R_{N}$ independent of $A, A_{1}, \ldots, A_{N}, B, B_{0}$ which solve (1.1) in law. Moreover, $\mathbb{E}\left(|R|^{s}\right)<\infty$ for every $s<s_{2}$.

Remark 1.8 The uniqueness of the solution of (1.1) will be explained in details in Sect. 3-see the discussion after Lemma 3.8. 
Section 3 contains a detailed proof of Theorem 1.7, which is similar in spirit to that of [13]. However, the multidimensional framework, we consider, provides some difficulties which do not appear in the one dimensional case. Namely, the method developed in [13], which gives finiteness of appropriate moments for the solution of (1.1), breaks down in higher dimensions. This problem will be dealt with the help of condition $(\mathcal{C})$ and additionally for technical reasons we have to assume that there is $s_{1} \leq \frac{1}{2}$ such that $\mathbb{E}\left(\|A\|^{S_{1}}\right) \leq \frac{1}{N}$.

The last condition allows us to give elementary proof of Theorem 1.7, which follows the ideas introduced in [13]. If we did not assume that $s_{1} \leq \frac{1}{2}$, it would generate many obstacles difficult to surmount. In particular straightforward proof of Lemma 3.12 which we propose in Sect. 3 might not work at all. On the one hand, the existence of $s_{1} \leq \frac{1}{2}$ such that $\mathbb{E}\left(\|A\|^{s_{1}}\right) \leq \frac{1}{N}$ can be relaxed in the one dimensional case (see [13-15]). On the other hand, it can be also relaxed in the multidimensional settings, but this requires more sophisticated techniques giving the existence of the solution of (1.1). This approach will be discussed in the forthcoming article of Buraczewski et al. [4].

Let $\lambda_{d}$ be the Lebesgue measure on $\mathbb{R}^{d}$. If $v$ is a probability measure on $\mathbb{R}^{d}$, then by $v=v_{a}+v_{s}$ we denote its Lebesgue decomposition with respect to $\lambda_{d}$ where $v_{a}$ is the absolutely continuous part with respect to $\lambda_{d}$, i.e. $v_{a} \ll \lambda_{d}$, and $v_{s}$ is the singular part with respect to $\lambda_{d}$, i.e. $v_{s} \perp \lambda_{d}$. We have also $v_{a} \perp v_{s}$. Since $v$ is positive then its total variation $\|v\|=v\left(\mathbb{R}^{d}\right)=1$. We say that the measure $v$ is singular if $\left\|v_{s}\right\|=1$, otherwise $v$ is nonsingular, i.e. $\left\|v_{s}\right\|<1$.

Now we can state our main "tail" result.

Theorem 1.9 Fix a natural number $N \geq 2$, a $G$-valued random matrix A distributed according to $\mu$, and a random vector $B$ with law $\eta$, independent of $A$, taking its values in $\mathbb{R}_{+}^{d}$, such that $\mathbb{P}(\{B>0\})>0$.

- Assume that $[$ supp $\mu] \subseteq G$ satisfies condition $(\mathcal{C})$, and there is $s_{1} \in(0,1 / 2]$, such that $\mathbb{E}\left(\|A\|^{s_{1}}\right)<\frac{1}{N}$. Moreover, we assume $s_{\infty}>s_{1}$ and $\lim _{s \rightarrow s_{\infty}} \kappa(s)>\frac{1}{N}$. Then there exists $\chi \geq s_{1}$ such that $N \kappa(\chi)=1$.

- Furthermore, if $\mathbb{E}\left(\|A\|^{\chi} \log ^{+}\|A\|\right)<\infty, E\left(|B|^{\chi+\varepsilon}\right)<\infty$ for some $\varepsilon>0$, and either

(i) $\eta$ is nonsingular, i.e. $\left\|\eta_{s}\right\|<1$, or

(ii) $\eta$ is singular, i.e. $\left\|\eta_{s}\right\|=1$, and $\mathbb{P}(\{\langle B, u\rangle=r\})=0$ for every $(u, r) \in$ $\mathbb{S}^{+} \times \mathbb{R}_{+}$.

Then there exists a positive function $e_{*}^{\chi}: \mathbb{S}^{+} \mapsto(0, \infty)$ and a constant $C_{\chi} \geq 0$ such that

$$
\lim _{t \rightarrow \infty} t^{\chi} \mathbb{P}(\{\langle R, u\rangle>t\})=C_{\chi} e_{*}^{\chi}(u) \geq 0,
$$

for every $u \in \mathbb{S}^{+}$, where $R \in \mathbb{R}_{+}^{d}$ is the stationary solution of $E q$. (1.1) as in Theorem 1.7. Moreover, if $\chi \geq 1$ then $C_{\chi}>0$, and the limit in (1.10) is strictly positive.

Now we give an example of singular measure $\eta$, i.e. $\left\|\eta_{s}\right\|=1$, on the plane $(d=2)$, such that $\eta\left(\left\{x \in \mathbb{R}^{2}:\langle x, u\rangle=r\right\}\right)=0$ for every $(u, r) \in \mathbb{S}^{+} \times \mathbb{R}_{+}$, and $\eta(\{x \in$ 
$\left.\left.\mathbb{R}^{2}: x>0\right\}\right)>0$. Define $S=\{(\cos \alpha, \sin \alpha): 0<\alpha<\pi / 2\} \subseteq \mathbb{S}^{+}$and let $\eta$ be the normalized one dimensional Lebesgue measure on $S$, i.e. $\operatorname{supp} \eta=\mathbb{S}^{+}$and $\eta(S)=1$. It is not hard to see that $\eta$ is singular with respect to two dimensional Lebesgue measure $\lambda_{2}$. Obviously $\eta\left(\left\{x \in \mathbb{R}^{2}: x>0\right\}\right)=\eta(S)=1$, and notice that $\left\{x \in \mathbb{R}^{2}:\langle x, u\rangle=r\right\}$ intersects $S$ at most two points, hence finally $\eta\left(\left\{x \in \mathbb{R}^{2}:\langle x, u\rangle=r\right\}\right)=0$.

As we mentioned before, the proof is based on concepts of [3] with considerable complications determined by the structure of Eq. (1.1). The most important tool which allows us to establish relation (1.10) is Kesten's renewal theorem [17]. We need to check that its assumptions are satisfied (see Sect. 4). This is the most difficult part of the paper and requires the spectral theory of transfer operators developed by Guivarc' $h$ and Le Page $[3,6,7]$, which is summarized in Sect. 2. But we touch only a few aspects of their theory and restrict our attention to the results which will be used in Sects. 3 and 4. Guivarc'h and Le Page approach significantly simplifies and clarifies proofs developed by Kesten [16], and what is most important for us, it is applicable to our situation.

The positivity of the limit constant $C_{\chi}>0$ in (1.10) if $\chi \geq 1$, is a very delicate issue. This relies strongly on the positivity of matrices and the fact that $\chi \geq 1$. In the case when $\chi<1$ the positivity of $C_{\chi}>0$ seems to be a very difficult problem and is unavailable in our situation at the moment. However, in the one dimensional case and the case of group of similarities (instead of group $G$ ), a very careful study (requiring complex analysis methods) of the formula defining the limit constant $C_{\chi} \geq 0$ allows us to conclude that the constant $C_{\chi}$ is nonzero. A detailed exposition of these, and related problems, are discussed in [4].

Remark 1.11 We would like to emphasize that there are some possible extensions of Theorems 1.7 and 1.9 which relax the assumption that $N \geq 2$ is constant and allows us to consider an integer-valued random variable $N \geq 2$ with appropriate moment conditions (see $[3,13-15,18]$ ). But this is not the main issue of this paper and therefore, for simplicity, we decided to assume that $N \geq 2$ is constant.

\section{Transfer operators}

Let $\mathcal{C}\left(\mathbb{S}^{+}\right)$be the space of continuous functions on $\mathbb{S}^{+}$with the supremum norm $|\cdot|_{\infty}$. $\mathcal{H}_{\varepsilon}=\left\{\phi \in \mathcal{C}\left(\mathbb{S}^{+}\right):\|\phi\|_{\varepsilon}=|\phi|_{\infty}+[\phi]_{\varepsilon}<\infty\right\}, \varepsilon \in(0,1]$ is the space of all $\varepsilon$-Hölder functions on $\mathbb{S}^{+}$with

$$
[\phi]_{\varepsilon}=\sup _{x \neq y} \frac{|\phi(x)-\phi(y)|}{|x-y|^{\varepsilon}} .
$$

Given a closed subset $V$ of $\mathbb{S}^{+}, M^{1}(V)$ denotes the set of all probability measures on $V$, endowed with the weak topology. We say that $U \subseteq \mathbb{S}^{+}$is a subspace of $\mathbb{S}^{+}$, if $U=V \cap \mathbb{S}^{+}$for some subspace $V \subseteq \mathbb{R}^{d}$. A measure $v \in M^{1}\left(\mathbb{S}^{+}\right)$is said to be proper if $v(U)=0$ for every subspace $U \varsubsetneqq \mathbb{S}^{+}$. Here and subsequently, $\Lambda(\Gamma)=\left\{\bar{v}_{a} \in \mathbb{S}^{+}: v_{a}\right.$ is the dominant eigenvector of $\left.a \in \Gamma^{\text {prox }}\right\}$, where $\Gamma$ is a subsemigroup of $G$ such that $\Gamma^{\text {prox }} \neq \varnothing$. 
The following Proposition 2.1 due to Guivarc' $h$ and Raugi [8] (see also [9]) contains the relevant properties of $(i-p)$ semigroups which will be used in the sequel.

Proposition 2.1 Let $\mu \in M^{1}(G)$ and $\Gamma=[$ supp $\mu]$ satisfies condition $(i-p)$. Then there exists a unique proper $\mu$-stationary measure $v \in M^{1}\left(\mathbb{S}^{+}\right)$such that suppv $=$ $\Lambda(\Gamma)$. Furthermore, $\Lambda(\Gamma)$ is the unique $\Gamma$-minimal subset of $\mathbb{S}^{+}$(i.e. if $Z \subseteq \mathbb{S}^{+}$is closed and $\Gamma \cdot Z \subseteq Z$, then $\Lambda(\Gamma) \subseteq Z$ ), and the subgroup of $\mathbb{R}_{+}^{*}$ generated by the set $\left\{\left|\lambda_{a}\right|: a \in \Gamma^{\text {prox }}\right\}$ is dense in $\mathbb{R}_{+}^{*}$.

Let $\mu \in M^{1}(G)$. For $s \in I_{\mu}, x \in \mathbb{S}^{+}$and a measurable function $\phi$ on $\mathbb{S}^{+}$we consider the following transfer operators

$$
\begin{aligned}
& P^{s} \phi(x)=\int_{G}|a x|^{s} \phi(a \cdot x) \mu(d a), \\
& P_{*}^{s} \phi(x)=\int_{G}\left|a^{*} x\right|^{s} \phi\left(a^{*} \cdot x\right) \mu(d a)=\int_{G}|a x|^{s} \phi(a \cdot x) \mu_{*}(d a),
\end{aligned}
$$

where $\mu_{*} \in M^{1}(G)$ and $\mu_{*}(U)=\mu\left(\left\{a \in G: a^{*} \in U\right\}\right)$ for every $U \in \mathcal{B} \operatorname{or}(G)$.

The main purpose of this section is to summarize a number of properties of operators $P^{s}, P_{*}^{s}$, see Theorem 2.3 below.

Theorem 2.3 Assume that $\mu \in M^{1}(G), s \in I_{\mu}$ and $\Gamma=[$ supp $\mu]$ satisfies condition $(i-p)$. Then

- there exists a unique probability measure $v^{s} \in M^{1}\left(\mathbb{S}^{+}\right),\left(v_{*}^{s} \in M^{1}\left(\mathbb{S}^{+}\right)\right)$such that

(i) $P^{s} v^{s}=\kappa(s) v^{s},\left(P_{*}^{s} v_{*}^{s}=\kappa(s) \nu_{*}^{s}\right)$.

(ii) $\operatorname{supp}^{s}=\Lambda([\operatorname{supp} \mu]),\left(\operatorname{supp} v_{*}^{s}=\Lambda\left(\left[\operatorname{supp} \mu_{*}\right]\right)\right)$ and it is not contained in any proper subspace of $\mathbb{S}^{+}$.

(iii) $I_{\mu} \ni s \mapsto v^{s} \in M^{1}\left(\mathbb{S}^{+}\right),\left(I_{\mu} \ni s \mapsto v_{*}^{s} \in M^{1}\left(\mathbb{S}^{+}\right)\right)$is continuous in the weak topology.

- $I_{\mu} \ni s \mapsto \kappa(s)$ is strictly log-convex function.

- there exists a unique $\underline{s}$-Hölder continuous function $e^{s}: \mathbb{S}^{+} \mapsto(0, \infty),\left(e_{*}^{s}: \mathbb{S}^{+} \mapsto\right.$ $(0, \infty))$ with $\underline{s}=\min \{s, 1\}$ such that

(i) $P^{s} e^{s}=\kappa(s) e^{s},\left(P_{*}^{s} e_{*}^{s}=\kappa(s) e_{*}^{s}\right)$.

(ii) $e^{s},\left(e_{*}^{s}\right)$ is given by the formula

$$
e^{s}(x)=\int_{\mathbb{S}^{+}}\langle x, y\rangle^{s} v_{*}^{s}(d y), \quad\left(e_{*}^{s}(x)=\int_{\mathbb{S}^{+}}\langle x, y\rangle^{s} v^{s}(d y)\right), \text { for } x \in \mathbb{S}^{+}
$$

(iii) $I_{\mu} \ni s \mapsto e^{s} \in \mathcal{C}\left(\mathbb{S}^{+}\right),\left(I_{\mu} \ni s \mapsto e_{*}^{s} \in \mathcal{C}\left(\mathbb{S}^{+}\right)\right)$is continuous in the uniform topology.

- Moreover, there exists a unique stationarymeasure $\pi^{s} \in M^{1}\left(\mathbb{S}^{+}\right),\left(\pi_{*}^{s} \in M^{1}\left(\mathbb{S}^{+}\right)\right)$ for operator $Q^{s} f=\frac{P^{s}\left(e^{s} f\right)}{\kappa(s) e^{s}},\left(Q_{*}^{s} f=\frac{P_{*}^{s}\left(e_{*}^{s} f\right)}{\kappa(s) e_{*}^{s}}\right)$ where $f \in \mathcal{C}\left(\mathbb{S}^{+}\right)$, such that

(i) $\pi^{s}=\frac{e^{s} v^{s}}{v^{s}\left(e^{s}\right)},\left(\pi_{*}^{s}=\frac{e_{*}^{s} v_{*}^{s}}{v_{*}^{s}\left(e_{*}^{s}\right)}\right)$. 
(ii) $\left(Q^{s}\right)^{n} f,\left(\left(Q_{*}^{s}\right)^{n} f\right)$ converges uniformly to $\pi^{s}(f),\left(\pi_{*}^{s}(f)\right)$ for any $f \in$ $\mathcal{C}\left(\mathbb{S}^{+}\right)$.

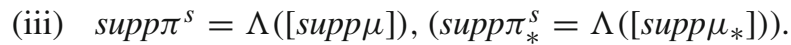

This result was proved by Guivarc'h and Le Page and its, quite long and far from being obvious, proof can be found in [6,7]. Notice that in view of the cocycle property $\sigma^{s}\left(x, a_{2} a_{1}\right)=\sigma^{s}\left(x, a_{1}\right) \sigma^{s}\left(a_{1} \cdot x, a_{2}\right),\left(\sigma_{*}^{s}\left(x, a_{2} a_{1}\right)=\sigma_{*}^{s}\left(x, a_{1}\right) \sigma_{*}^{s}\left(a_{1} \cdot x, a_{2}\right)\right), a_{1}$, $a_{2} \in G, x \in \mathbb{S}^{+}$of

$$
\sigma^{s}(x, a)=|a x|^{s} \frac{e^{s}(a \cdot x)}{e^{s}(x)}, \quad\left(\sigma_{*}^{s}(x, a)=|a x|^{s} \frac{e_{*}^{s}(a \cdot x)}{e_{*}^{s}(x)}\right),
$$

the Markov operators $Q^{S}$ and $Q_{*}^{S}$ defined in Theorem 2.3 can be rewritten in the following form

$$
\begin{aligned}
& \left(Q^{s}\right)^{n} \phi(x)=\int_{G} \phi(a \cdot x) q_{n}^{s}(x, a) \mu^{* n}(d a), \\
& \left(Q_{*}^{s}\right)^{n} \phi(x)=\int_{G} \phi(a \cdot x) q_{n}^{s, *}(x, a) \mu_{*}^{* n}(d a),
\end{aligned}
$$

where

$$
\begin{aligned}
q_{n}^{s}(x, a) & =\frac{1}{\kappa^{n}(s)} \frac{e^{s}(a \cdot x)}{e^{s}(x)}|a x|^{s}=\frac{\sigma^{s}(x, a)}{\kappa^{n}(s)}, \\
q_{n}^{s, *}(x, a) & =\frac{1}{\kappa^{n}(s)} \frac{e_{*}^{s}(a \cdot x)}{e_{*}^{s}(x)}|a x|^{s}=\frac{\sigma_{*}^{s}(x, a)}{\kappa^{n}(s)},
\end{aligned}
$$

$n \in \mathbb{N}, x \in \mathbb{S}^{+}, a \in G$ and $\phi$ is an arbitrary measurable function on $\mathbb{S}^{+}$.

\section{Construction of the solution}

Recall that $A$ stands for a $G$-valued random matrix distributed according to the measure $\mu \in M^{1}(G)$, and $B$ for a random vector taking its values in $\mathbb{R}_{+}^{d}$, independent of $A$. In this section we construct a solution of Eq. (1.1). The idea of the construction goes back to [13]. It is not difficult to imagine that we have to study a sequence of random variables that are obtained by iterating (1.1). Let $N \geq 2$ be a fixed natural number and $R_{0,1}^{*}, \ldots, R_{0, N}^{*}$ be independent and identically distributed (i.i.d.) copies of the initial random variable $R_{0}^{*} \in \mathbb{R}_{+}^{d}$. Throughout the paper we will assume that $\mathbb{E}\left(\left|R_{0}^{*}\right|^{s_{2}}\right)<\infty$, for $s_{2}>0$ as in Theorem 1.7. We consider the sequence $\left(R_{n}^{*}\right)_{n \geq 0}$ such that

$$
R_{n+1}^{*}=\sum_{k=1}^{N} A_{n+1, k} R_{n, k}^{*}+B_{n+1}, \quad \text { for every } n \geq 0,
$$


where $A_{n+1,1}, \ldots, A_{n+1, N}, B_{n+1}$ and $R_{n, 1}^{*}, \ldots, R_{n, N}^{*}, n \geq 0$ are independent. Moreover, for $n \geq 1 R_{n, 1}^{*}, \ldots, R_{n, N}^{*}$ are i.i.d. copies of $R_{n}^{*}$ obtained at the previous iteration. For $n \geq 0 A_{n+1,1}, \ldots, A_{n+1, N}$ are i.i.d. copies of $A$ and $B_{n+1}$ is an independent copy of $B$.

We will look more closely at the sequence $\left(R_{n}^{*}\right)_{n \geq 0}$. Let $\mathcal{A}=\left\{A_{i_{1}, \ldots, i_{n}}\right.$ : $\left.\left(i_{1}, \ldots, i_{n}\right) \in\{1, \ldots, N\}^{n}, n \in \mathbb{N}\right\}$ be the set consisting of i.i.d. copies of $A$, and $\mathcal{B}=\left\{B_{i_{1}, \ldots, i_{n}}:\left(i_{1}, \ldots, i_{n}\right) \in\{1, \ldots, N\}^{n}, n \in \mathbb{N}\right\} \cup\left\{B_{0}\right\}$ the set consisting of i.i.d. copies of $B$ independent of $\mathcal{A}$. Additionally we assume that $A_{0}=$ Id a.s. and the initial random variable $R_{0}^{*}$ is always independent of $A, B, \mathcal{A}$ and $\mathcal{B}$.

Now let $W_{0}=A_{0} B_{0}=B_{0}$ a.s.,

$$
W_{n}=\sum_{\left(i_{1}, \ldots, i_{n}\right) \in\{1, \ldots, N\}^{n}} A_{i_{1}} A_{i_{1}, i_{2}} \cdots A_{i_{1}, \ldots, i_{n}} B_{i_{1}, \ldots, i_{n}}, \quad n \geq 1
$$

and for $n \geq 0$

$$
R^{(n)}=\sum_{i=0}^{n} W_{i}
$$

be the partial sum of the sequence $\left(W_{n}\right)_{n \geq 0}$. Since $R^{(n+1)}-R^{(n)} \geq 0$ is a positive vector for every $n \in \mathbb{N}$ then

$$
R=\lim _{n \rightarrow \infty} R^{(n)}=\sum_{i=0}^{\infty} W_{i}
$$

exists a.s. and is a candidate for a solution of (1.1). Indeed, it is not hard to see that $W_{n}$ satisfies

$$
\begin{aligned}
W_{n} & =\sum_{\left(i_{1}, \ldots, i_{n}\right) \in\{1, \ldots, N\}^{n}} A_{i_{1}} A_{i_{1}, i_{2}} \cdots A_{i_{1}, \ldots, i_{n}} B_{i_{1}, \ldots, i_{n}} \\
& =\sum_{k=1}^{N} A_{k}\left(\sum_{\left(k, i_{2}, \ldots, i_{n}\right) \in\{1, \ldots, N\}^{n}} A_{k, i_{2}} \cdots A_{k, i_{2}, \ldots, i_{n}} B_{k, i_{2}, \ldots, i_{n}}\right)=\sum_{k=1}^{N} A_{k} W_{n-1, k},
\end{aligned}
$$

where $A_{k}$ and $W_{n-1, k}$ are independent of each other and $W_{n-1,1}, \ldots, W_{n-1, N}$ have the same distribution as $W_{n-1}$. In view of the above calculations, $R^{(n)}$ satisfies the recursion

$$
R^{(n)}=\sum_{k=1}^{N} A_{k} R_{k}^{(n-1)}+B_{0}
$$


for every $n \in \mathbb{N}$, where $R_{1}^{(n-1)}, \ldots, R_{N}^{(n-1)}$ are independent copies of $R^{(n-1)}$. This allows us to conclude that $R$ is a solution of (1.1) in law provided that $R$ is finite a.s., but this will be shown in the proof of Theorem 1.7 below.

To obtain a solution with an initial condition, let $R_{0,\left(i_{1}, \ldots, i_{n}\right)}^{*},\left(i_{1}, \ldots, i_{n}\right) \in$ $\{1, \ldots, N\}^{n}, n \in \mathbb{N}$, be i.i.d. copies of the initial random variable $R_{0}^{*} \in \mathbb{R}_{+}^{d}$ independent of the families $\mathcal{A}$ and $\mathcal{B}$. For $n \geq 1$, we define similarly as in (3.2)

$$
W_{n}\left(R_{0}^{*}\right)=\sum_{\left(i_{1}, \ldots, i_{n}\right) \in\{1, \ldots, N\}^{n}} A_{i_{1}} A_{i_{1}, i_{2}} \cdots A_{i_{1}, \ldots, i_{n}} R_{0,\left(i_{1}, \ldots, i_{n}\right)}^{*}
$$

Moreover, as in (3.5), we obtain $W_{n}\left(R_{0}^{*}\right) \stackrel{\mathcal{D}}{=} \sum_{k=1}^{N} A_{k} W_{n-1, k}\left(R_{0}^{*}\right)$, where $A_{k}$ and $W_{n-1, k}\left(R_{0}^{*}\right)$ are independent of each other and $W_{n-1,1}\left(R_{0}^{*}\right), \ldots, W_{n-1, N}\left(R_{0}^{*}\right)$ have the same distribution as $W_{n-1}\left(R_{0}^{*}\right)$. Now we have the following

Lemma 3.8 Assume now that $\left(R_{n}^{*}\right)_{n \geq 0}$ and $\left(R^{(n)}\right)_{n \geq 0}$ are the sequences defined in (3.1) and (3.3), respectively, then for every $n \in \mathbb{N}$ we have

$$
R_{n}^{*} \stackrel{\mathcal{D}}{=} R^{(n-1)}+W_{n}\left(R_{0}^{*}\right)
$$

Proof Observe that for $n=1$, (3.9) follows from definition. For more details we refer to [13].

In view of formula (3.9) we will be able to show (in the proof of Theorem 1.7 below) that every sequence $\left(R_{n}^{*}\right)_{n \geq 0}$ obtained from the iterations described at the beginning of Sect. 3 (see (3.1)) converges in law to the random variable $R$ defined in (3.4), provided that $\mathbb{E}\left(\left|R_{0}^{*}\right|^{S_{2}}\right)<\infty$. The uniqueness of the solution of (1.1) will be understood exactly in the sense described above. Therefore, one may think that the solution of (1.1) does not depend on the choice of the initial random variable $R_{0}^{*}$.

Now we have the simple, but very useful

Lemma 3.10 Under the assumptions of Theorem 2.3 there exists $c_{s}>0$ such that for every $n \in \mathbb{N}$ we have

$$
c_{s} \int_{G}\|a\|^{s} \mu^{n}(d a) \leq \kappa^{n}(s) \leq \int_{G}\|a\|^{s} \mu^{n}(d a) .
$$

Proof We refer to [6]. 
To take the limit in (3.4) we need an estimate for $\mathbb{E}\left(\left|W_{n}\right|^{s}\right)$. Suppose for a moment that $s \leq 1$. Then, in view of inequality (3.11), we have

$$
\begin{aligned}
\mathbb{E}\left(\left|W_{n}\right|^{s}\right) & \leq \mathbb{E}\left(\sum_{\left(i_{1}, \ldots, i_{n}\right) \in\{1, \ldots, N\}^{n}}\left\|A_{i_{1}} A_{i_{1}, i_{2}} \cdots A_{i_{1}, \ldots, i_{n}}\right\|^{s}\left|B_{i_{1}, \ldots, i_{n}}\right|^{s}\right) \\
& \leq \sum_{\left(i_{1}, \ldots, i_{n}\right) \in\{1, \ldots, N\}^{n}} \mathbb{E}\left(\left\|A_{i_{1}} A_{i_{1}, i_{2}} \cdots A_{i_{1}, \ldots, i_{n}}\right\|^{s}\right) \mathbb{E}\left(|B|^{s}\right) \\
& =N^{n} \int_{G}\|a\|^{s} \mu^{* n}(d a) \mathbb{E}\left(|B|^{s}\right) \leq \frac{1}{c_{s}} \mathbb{E}\left(|B|^{s}\right) N^{n} \kappa^{n}(s) .
\end{aligned}
$$

We would like to show that for an appropriate $s>0$, not necessarily less or equal 1 , the quantity $\mathbb{E}\left(\left|W_{n}\right|^{s}\right)$ decays exponentially. This is contained in Lemma 3.12. For the sake of computations we have to assume that there exists $s_{1} \in(0,1 / 2]$ such that $\mathbb{E}\left(\|A\|^{s_{1}}\right) \leq \frac{1}{N}$.

Lemma 3.12 Assume that $[$ supp $\mu] \subseteq G$ satisfies condition $(\mathcal{C})$, and there exist $s_{1} \in$ $(0,1 / 2]$, and $s_{2}>1$ such that $\mathbb{E}\left(\|A\|^{s_{1}}\right) \leq \frac{1}{N}, \mathbb{E}\left(\|A\|^{s_{2}}\right) \leq \frac{1}{N}$, and $\mathbb{E}\left(|B|^{s_{2}}\right)<\infty$. Then for every $s \in\left(s_{1}, s_{2}\right)$, there exist finite constants $K_{s}>0$ and $\eta<1$ such that for every $n \in \mathbb{N}$

$$
\mathbb{E}\left(\left|W_{n}\right|^{s}\right) \leq K_{s} \eta^{n}
$$

Proof By Theorem $2.3 \kappa(s)$ is strictly log-convex so $N \kappa(s)<1$, for every $s \in$ $\left(s_{1}, s_{2}\right)$ and for $s \leq 1$, (3.13) follows from the calculation above. From now we assume that $s \in\left(1, s_{2}\right)$ and it is fixed. Let $S_{i_{1}, \ldots, i_{n}}=A_{i_{1}} A_{i_{1}, i_{2}} \cdots A_{i_{1}, \ldots, i_{n}}$ for $\left(i_{1}, \ldots, i_{n}\right) \in\{1, \ldots, N\}^{n}$ and $n \in \mathbb{N}$. We order the set of indices writing $\{1, \ldots, N\}^{n}=\left\{\mathbf{i}_{1}, \ldots, \mathbf{i}_{N^{n}}\right\}$ and we choose $p \in \mathbb{N}$ and $p \geq 2$, such that $p-1<$ $s \leq p$. Then $s_{1} \leq 1 / 2<s / p \leq 1$ (here is the first time where we have used that $s_{1} \leq \frac{1}{2}$, this allows us to make the specific choice of $p$, which in turn guarantees that $k s / p \in\left(s_{1}, s_{2}\right)$ for every $k \in\{1,2, \ldots, p\}$ and the inequalities $N \kappa(k s / p)<1$, hold for every $k \in\{1,2, \ldots, p\})$. Moreover

$$
\begin{aligned}
& \mathbb{E}\left(\left|W_{n}\right|^{S}\right) \\
& \leq \mathbb{E}\left(\left(\sum_{\left(i_{1}, \ldots, i_{n}\right) \in\{1, \ldots, N\}^{n}}\left|S_{i_{1}, \ldots, i_{n}} B_{i_{1}, \ldots, i_{n} \mid}\right|^{s / p}\right)^{p}\right) \\
& =\mathbb{E}\left(\sum_{j_{\mathbf{i}_{1}}+\cdots+j_{\mathbf{i}_{N} n}=p}\left(\begin{array}{c}
p \\
j_{\mathbf{i}_{1}}, \ldots, j_{\mathbf{i}_{N^{n}}}
\end{array}\right)\left|S_{\mathbf{i}_{1}} B_{\mathbf{i}_{1}}\right|^{s j_{\mathbf{i}_{1}} / p \ldots} \ldots\left|S_{\mathbf{i}_{N^{n}}} B_{\mathbf{i}_{N^{n}}}\right|^{s j_{\mathbf{i}_{N}} / p}\right) \\
& \leq \sum_{j_{\mathbf{i}_{1}}+\cdots+j_{\mathbf{i}_{N^{n}}}=p}\left(\begin{array}{c}
p \\
j_{\mathbf{i}_{1}}, \ldots, j_{\mathbf{i}_{N^{n}}}
\end{array}\right) \mathbb{E}\left(\left(\left\|S_{\mathbf{i}_{1}}\right\|\left|B_{\mathbf{i}_{1}}\right|\right)^{s j j_{\mathbf{i}_{1}} / p}\right) \cdots \mathbb{E}\left(\left(\left\|S_{\mathbf{i}_{N^{n}}}\right\|\left|B_{\mathbf{i}_{N^{n}}}\right|\right)^{s j j_{\mathbf{i}^{n}} / p}\right) .
\end{aligned}
$$


Notice that $\mathbb{E}\left(\left|B_{\mathbf{i}_{1}}\right|^{s j \dot{\mathbf{i}}_{1} / p}\right) \cdots \mathbb{E}\left(\left|B_{\mathbf{i}_{N^{n}}}\right|^{s j \mathbf{i}_{N^{n}} / p}\right)=\|B\|_{s j_{\mathbf{i}_{1}} / p}^{s j j_{\mathbf{i}_{1}} / p} \cdots\|B\|_{s j_{\mathbf{i}^{n}} / p}^{s j \mathbf{i}_{N^{n}} / p} \leq$ $\|B\|_{s}^{s}$, since $\|B\|_{r}=\mathbb{E}\left(|B|^{r}\right)^{1 / r}$ is increasing and $\|B\|_{0}=1$. This implies that

$$
\begin{aligned}
& \sum_{j_{\mathbf{i}_{1}}+\cdots+j_{\mathbf{i}^{n}}=p}\left(\begin{array}{c}
p \\
j_{\mathbf{i}_{1}}, \ldots, j_{\mathbf{i}_{N^{n}}}
\end{array}\right) \mathbb{E}\left(\left(\left\|S_{\mathbf{i}_{1}}\right\|\left|B_{\mathbf{i}_{1}}\right|\right)^{s j_{\mathbf{i}_{1}} / p}\right) \cdots \mathbb{E}\left(\left(\left\|S_{\mathbf{i}_{N^{n}}}\right\|\left|B_{\mathbf{i}_{N^{n}}}\right|\right)^{s \dot{\mathbf{i}}_{N^{n}} / p}\right) \\
& \leq \mathbb{E}\left(|B|^{s}\right) \sum_{j_{\mathbf{i}_{1}}+\cdots+j_{\mathbf{i}_{N^{n}}}=p}\left(\begin{array}{c}
p \\
j_{\mathbf{i}_{1}}, \ldots, j_{\mathbf{i}_{N^{n}}}
\end{array}\right) \mathbb{E}\left(\left\|S_{\mathbf{i}_{1}}\right\|^{s \mathbf{i}_{1} / p}\right) \cdots \mathbb{E}\left(\left\|S_{\mathbf{i}_{N^{n}}}\right\|^{s \mathbf{i}_{N^{n}} / p}\right) \\
& =\mathbb{E}\left(|B|^{s}\right) \sum_{j_{\mathbf{i}_{1}}+\cdots+j_{\mathbf{i}_{N^{n}}}=p}\left(\begin{array}{c}
p \\
j_{\mathbf{i}_{1}}, \ldots, j_{\mathbf{i}_{N} n}
\end{array}\right) \int_{G}\|a\|^{s j_{\mathbf{i}_{1}} / p} \mu^{* n}(d a) \cdots \int_{G}\|a\|^{s \mathbf{i}_{N^{n}} / p} \mu^{* n}(d a) .
\end{aligned}
$$

Observe that by the inequality (3.11), there exist constants $c_{s j_{\mathbf{i}_{1}} / p}, c_{s j_{\mathbf{i}_{2}} / p}, \ldots$, $c_{S j_{\mathbf{i}^{n}} / p} \in(0,1]$, such that for all $n \in \mathbb{N}$

$$
\begin{aligned}
& \int_{G}\|a\|^{s j_{\mathbf{i}_{1}} / p} \mu^{* n}(d a) \leq c_{s j_{\mathbf{i}_{1}} / p}^{-1} \kappa^{n}\left(s j_{\mathbf{i}_{1}} / p\right), \\
& \int_{G}\|a\|^{s j_{\mathbf{i}_{2}} / p} \mu^{* n}(d a) \leq c_{s j_{\mathbf{i}_{2}} / p}^{-1} \kappa^{n}\left(s j_{\mathbf{i}_{2}} / p\right), \\
& \int_{G}\|a\|^{s j_{\mathbf{i}_{N}} / p} \mu^{* n}(d a) \leq c_{s j_{\mathbf{i}_{N}}}^{-1} / p \kappa^{n}\left(s j_{\mathbf{i}_{N^{n}}} / p\right) .
\end{aligned}
$$

Since $j_{\mathbf{i}_{1}}, j_{\mathbf{i}_{2}}, \ldots, j_{\mathbf{i}_{N^{n}}} \in\{0,1, \ldots, p\}$, the constants above do not depend on $n \in \mathbb{N}$ and we may define $c_{p, s}=\max \left\{c_{0}^{-1}, c_{s / p}^{-1}, c_{2 s / p}^{-1}, \ldots, c_{(p-1) s / p}^{-1}, c_{s}^{-1}\right\}$ that dominates all of them.

When $N^{n} \leq p$, we have

$$
\int_{G}\|a\|^{s j_{\mathbf{i}_{1}} / p} \mu^{* n}(d a) \cdots \int_{G}\|a\|^{s j_{\mathbf{i}^{n}} / p} \mu^{* n}(d a) \leq c_{p, s}^{p} \kappa^{n}\left(s j_{\mathbf{i}_{1}} / p\right) \cdots \cdots \kappa^{n}\left(s j_{\mathbf{i}_{N^{n}}} / p\right) .
$$

Therefore,

$$
\begin{aligned}
& \sum_{j_{\mathbf{i}_{1}}+\cdots+j_{\mathbf{i}_{N^{n}}}=p}\left(\begin{array}{c}
p \\
j_{\mathbf{i}_{1}}, \ldots, j_{\mathbf{i}_{N^{n}}}
\end{array}\right) \int_{G}\|a\|^{s j_{\mathbf{i}_{1}} / p} \mu^{* n}(d a) \cdots \int_{G}\|a\|^{s \mathbf{i}_{N^{n}} / p} \mu^{* n}(d a) \\
\leq & c_{p, s}^{p} \sum_{j_{\mathbf{i}_{1}+\cdots+j_{\mathbf{i}^{n}}=p}}\left(\begin{array}{c}
p \\
j_{\mathbf{i}_{1}}, \ldots, j_{\mathbf{i}_{N^{n}}}
\end{array}\right) \kappa^{n}\left(s j_{\mathbf{i}_{1}} / p\right) \cdots \kappa^{n}\left(s j_{\mathbf{i}_{N^{n}}} / p\right) \\
\leq & c_{p, s}^{p} \cdot \max \{\kappa(s / p), \kappa(2 s / p), \ldots, \kappa((p-1) s / p), \kappa(s)\}^{n}
\end{aligned}
$$




$$
\begin{aligned}
& \sum_{j_{\mathbf{i}_{1}}+\ldots+j_{\mathbf{i}_{N^{n}}}=p}\left(\begin{array}{c}
p \\
j_{\mathbf{i}_{1}}, \ldots, j_{\mathbf{i}_{N^{n}}}
\end{array}\right) \\
& \leq c_{p, s}^{p} N^{p n} \cdot \max \{\kappa(s / p), \kappa(2 s / p), \ldots, \kappa((p-1) s / p), \kappa(s)\}^{n} \\
& \leq c_{p, s}^{p} p^{p-1} N^{n} \cdot \max \{\kappa(s / p), \kappa(2 s / p), \ldots, \kappa((p-1) s / p), \kappa(s)\}^{n},
\end{aligned}
$$

since $k s / p \in\left(s_{1}, s_{2}\right)$ for every $k \in\{1, \ldots, p\}$. This yields (3.13) with $K_{s}=$ $c_{p, s}^{p} p^{p-1} \mathbb{E}\left(|B|^{s}\right)<\infty$ and $\eta=N \cdot \max \{\kappa(s / p), \ldots, \kappa(s)\}<1$. As we said before the assumption $s_{1} \leq 1 / 2$ is indispensable, because it guarantees that $N \cdot \kappa(k s / p)<1$ for every $k \in\{1,2, \ldots, p\}$.

When $N^{n}>p,(3.14)$ also holds with the universal constant $c_{p, s}^{p}$ which does not depend on $n \in \mathbb{N}$, but we have to estimate

$$
\sum_{j_{\mathbf{i}_{1}}+\cdots+j_{\mathbf{i}_{N}}=p}\left(\begin{array}{c}
p \\
j_{\mathbf{i}_{1}}, \ldots, j_{\mathbf{i}_{N^{n}}}
\end{array}\right) \kappa^{n}\left(s j_{\mathbf{i}_{1}} / p\right) \cdots \kappa^{n}\left(s j_{\mathbf{i}_{N^{n}}} / p\right),
$$

in a more subtle way. Before we do that we need to introduce a portion of necessary definitions.

For every $r \leq k$, and $j_{1} \leq \cdots \leq j_{k}$, let

$$
L\left(j_{1}, \ldots, j_{k}\right)=\left(\begin{array}{c}
k \\
l_{1}, l_{2}, \ldots, l_{r}
\end{array}\right)
$$

when $j_{1}=\cdots=j_{l_{1}}<j_{l_{1}+1}=\cdots=j_{l_{2}+l_{1}}<j_{l_{2}+l_{1}+1}=\cdots=j_{l_{3}+l_{2}+l_{1}}<\cdots<$ $j_{l_{r-1}+\cdots+l_{1}+1}=\cdots=j_{l_{r}+\cdots+l_{1}}$ and $l_{1}+l_{2}+\cdots+l_{r}=k$. Then it is not difficult to see that for every $k \leq p$

$$
\begin{aligned}
L\left(j_{1}, \ldots, j_{k}\right) & \leq k ! \\
\left(\begin{array}{c}
p \\
j_{1}, \ldots, j_{k}
\end{array}\right) & \leq p ! \\
\left(\begin{array}{c}
N^{n} \\
k
\end{array}\right) L\left(j_{1}, \ldots, j_{k}\right) & \leq \frac{N^{n} !}{\left(N^{n}-k\right) !} \leq N^{k n} .
\end{aligned}
$$

Let now $\eta=\max \left\{\eta_{1}, \eta_{2}, \ldots, \eta_{p}\right\}<1$, where

$$
\begin{aligned}
& \eta_{k}=\max \left\{\left(N \kappa\left(s j_{1} / p\right)\right) \cdots\left(N \kappa\left(s j_{k} / p\right)\right): j_{1}+\cdots+j_{k}=p,\right. \text { and } \\
& \left.j_{1} \leq \cdots \leq j_{k}\right\}<1 .
\end{aligned}
$$

This implies that

$$
\sum_{j_{\mathbf{i}_{1}}+\cdots+j_{\mathbf{i}_{N^{n}}}=p}\left(\begin{array}{c}
p \\
j_{\mathbf{i}_{1}}, \ldots, j_{\mathbf{i}_{N^{n}}}
\end{array}\right) \kappa^{n}\left(s j_{\mathbf{i}_{1}} / p\right) \cdots \cdots \kappa^{n}\left(s j_{\mathbf{i}_{N^{n}}} / p\right)=N^{n} \kappa^{n}(s)
$$




$$
\begin{aligned}
& +\left(\begin{array}{c}
N^{n} \\
2
\end{array}\right) \sum_{\substack{j_{1}+j_{2}=p \\
j_{1} \leq j_{2}}}\left(\begin{array}{c}
p \\
j_{1}, j_{2}
\end{array}\right) L\left(j_{1}, j_{2}\right) \kappa^{n}\left(s j_{1} / p\right) \kappa^{n}\left(s j_{2} / p\right) \\
& +\left(\begin{array}{c}
N^{n} \\
3
\end{array}\right) \sum_{\substack{j_{1}+j_{2}+j_{3}=p \\
j_{1} \leq j_{2} \leq j_{3}}}\left(\begin{array}{c}
p \\
j_{1}, j_{2}, j_{3}
\end{array}\right) L\left(j_{1}, j_{2}, j_{3}\right) \kappa^{n}\left(s j_{1} / p\right) \kappa^{n}\left(s j_{2} / p\right) \kappa^{n}\left(s j_{3} / p\right) \\
& +\left(\begin{array}{c}
N^{n} \\
k
\end{array}\right) \sum_{\substack{j_{1}+\cdots+j_{k}=p \\
j_{1} \leq \cdots \leq j_{k}}}\left(\begin{array}{c}
p \\
j_{1}, \ldots, j_{k}
\end{array}\right) L\left(j_{1}, \ldots, j_{k}\right) \kappa^{n}\left(s j_{1} / p\right) \cdots \kappa^{n}\left(s j_{k} / p\right) \\
& +\left(\begin{array}{c}
N^{n} \\
p
\end{array}\right) \sum_{\substack{j_{1}+\cdots+j_{p}=p \\
j_{1} \leq \cdots \leq j p}}\left(\begin{array}{c}
p \\
j_{1}, \ldots, j_{p}
\end{array}\right) L\left(j_{1}, \ldots, j_{p}\right) \kappa^{n}\left(s j_{1} / p\right) \cdots \kappa^{n}\left(s j_{p} / p\right) \\
& \leq N^{n} \kappa^{n}(s)+\sum_{\substack{j_{1}+j_{2}=p \\
j_{1} \leq j_{2}}} p ! N^{2 n} \kappa^{n}\left(s j_{1} / p\right) \kappa^{n}\left(s j_{2} / p\right) \\
& +\cdots+\sum_{\substack{j_{1}+\cdots+j_{k}=p \\
j_{1} \leq \cdots \leq j_{k}}} p ! N^{k n} \kappa^{n}\left(s j_{1} / p\right) \cdots \cdots \kappa^{n}\left(s j_{k} / p\right) \\
& +\cdots+\sum_{\substack{j_{1}+\cdots+j_{p}=p \\
j_{1} \leq \cdots \leq j p}} p ! N^{p n} \kappa^{n}\left(s j_{1} / p\right) \cdots \cdots \kappa^{n}\left(s j_{p} / p\right) \leq \eta^{n} \cdot p ! \sum_{k=1}^{p} \sum_{\substack{j_{1}+\cdots+j_{k}=p \\
j_{1} \leq \cdots \leq j_{k}}} 1 \\
& \leq \eta^{n} \cdot p ! \sum_{k=1}^{p}\left(\begin{array}{l}
p-1 \\
k-1
\end{array}\right) \leq 2^{p-1} p ! \cdot \eta^{n}
\end{aligned}
$$

Hence in this case (3.13) follows with $K_{s}=2^{p-1} p ! c_{p, s}^{p} \mathbb{E}\left(|B|^{s}\right)<\infty$ and $\eta<1$.

Proof of Theorem 1.7 First of all we show that $\mathbb{E}\left(|R|^{s}\right)<\infty$ for every $s<s_{2}$, where $R$ was defined in (3.4). This shows that $R$ is finite a.s. moreover, in view of formula (3.6) $R$ solves Eq. (1.1) in law. Its uniqueness will be a consequence of Lemma 3.8 as we mentioned above.

By Lemma 3.12 there exist $\eta<1$ and $K_{s}<\infty$ such that for every $n \in \mathbb{N}$ we have $\mathbb{E}\left(\left|W_{n}\right|^{s}\right) \leq K_{s} \eta^{n}$. Observe now

$$
\mathbb{E}\left(|R|^{s}\right)=\mathbb{E}\left(\liminf _{n \rightarrow \infty}\left|R^{(n)}\right|^{s}\right) \leq \liminf _{n \rightarrow \infty} \mathbb{E}\left(\left|R^{(n)}\right|^{s}\right) \leq \liminf _{n \rightarrow \infty} \mathbb{E}\left(\sum_{k=0}^{n}\left|W_{k}\right|\right)^{s} .
$$

When $0<s \leq 1$, we have

$\liminf _{n \rightarrow \infty} \mathbb{E}\left(\sum_{k=0}^{n}\left|W_{k}\right|\right)^{s} \leq \liminf _{n \rightarrow \infty} \mathbb{E}\left(\sum_{k=0}^{n}\left|W_{k}\right|^{s}\right) \leq \liminf _{n \rightarrow \infty} K_{s} \sum_{k=0}^{n} \eta^{k}=\frac{K_{s}}{1-\eta}<\infty$ 
When $s>1$, we have

$$
\begin{aligned}
\liminf _{n \rightarrow \infty} \mathbb{E}\left(\sum_{k=0}^{n}\left|W_{k}\right|\right)^{s} & \leq \liminf _{n \rightarrow \infty}\left(\sum_{k=0}^{n} \mathbb{E}\left(\left|W_{k}\right|^{s}\right)^{1 / s}\right)^{s} \\
& \leq \liminf _{n \rightarrow \infty} K_{s}\left(\sum_{k=0}^{n} \eta^{k / s}\right)^{s}=\frac{K_{s}}{\left(1-\eta^{1 / s}\right)^{s}}<\infty
\end{aligned}
$$

It immediately implies that $\mathbb{E}\left(|R|^{s}\right)<\infty$, which in turn gives $|R|<\infty$ a.s.

Now we want to show that $R$ is the unique solution of (1.1). It is enough to show that $R_{n}^{*}$, with an arbitrary initial random variable $R_{0}^{*} \in \mathbb{R}_{+}^{d}$ converges weakly to $R$ as $n \rightarrow \infty$. Recall that the initial random variable $R_{0}^{*}$ has finite $s_{2}$ th moment, i.e. $\mathbb{E}\left(\left|R_{0}^{*}\right|^{S_{2}}\right)<\infty$. We show that $\mathbb{E}\left(f\left(R_{n}^{*}\right)\right) \underset{n \rightarrow \infty}{\longrightarrow} \mathbb{E}(f(R))$ for an arbitrary uniformly continuous function $f$ defined on $\mathbb{R}^{d}$. Fix $\varepsilon>0$, and choose $\delta>0$ such that

$$
|x-y|<\delta \Longrightarrow|f(x)-f(y)|<\varepsilon \text {. }
$$

By (3.9) we know that $R_{n}^{*} \stackrel{\mathcal{D}}{=} R^{(n-1)}+W_{n}\left(R_{0}^{*}\right)$ for every $n \in \mathbb{N}$, hence

$$
\begin{aligned}
\left|\mathbb{E}\left(f\left(R_{n}^{*}\right)-f(R)\right)\right| & \leq\left|\mathbb{E}\left(f\left(R^{(n-1)}+W_{n}\left(R_{0}^{*}\right)\right)-f\left(R^{(n-1)}\right)\right)\right| \\
& +\left|\mathbb{E}\left(f\left(R^{(n-1)}\right)-f(R)\right)\right|
\end{aligned}
$$

It is enough to show that $\left|\mathbb{E}\left(f\left(R^{(n-1)}+W_{n}\left(R_{0}^{*}\right)\right)-f\left(R^{(n-1)}\right)\right)\right| \underset{n \rightarrow \infty}{\longrightarrow} 0$. Fix $s<s_{2}$ and observe that, in view of inequality (3.13) with $W_{n}\left(R_{0}^{*}\right)$ instead of $W_{n}$, we have

$$
\begin{aligned}
& \left|\mathbb{E}\left(f\left(R^{(n-1)}+W_{n}\left(R_{0}^{*}\right)\right)-f\left(R^{(n-1)}\right)\right)\right| \\
& \quad \leq \mathbb{E}\left(\left|\mathbf{1}_{\left\{\left|W_{n}\left(R_{0}^{*}\right)\right| \leq \delta\right\}}\left(f\left(R^{(n-1)}+W_{n}\left(R_{0}^{*}\right)\right)-f\left(R^{(n-1)}\right)\right)\right|\right) \\
& \quad+\mathbb{E}\left(\left|\mathbf{1}_{\left\{\left|W_{n}\left(R_{0}^{*}\right)\right|>\delta\right\}}\left(f\left(R^{(n-1)}+W_{n}\left(R_{0}^{*}\right)\right)-f\left(R^{(n-1)}\right)\right)\right|\right) \\
& \quad \leq \varepsilon \mathbb{P}\left(\left\{\left|W_{n}\left(R_{0}^{*}\right)\right| \leq \delta\right\}\right)+2 M_{f} \mathbb{P}\left(\left\{\left|W_{n}\left(R_{0}^{*}\right)\right|>\delta\right\}\right) \\
& \quad \leq \varepsilon+2 M_{f} \mathbb{P}\left(\left\{\left|W_{n}\left(R_{0}^{*}\right)\right|>\delta\right\}\right) \leq \varepsilon+2 M_{f} \frac{\mathbb{E}\left(\left|W_{n}\left(R_{0}^{*}\right)\right|^{s}\right)}{\delta^{s}} \\
& \quad \leq \varepsilon+\frac{2 M_{f} K_{s}}{\delta^{s}} \eta^{n} \underset{n \rightarrow \infty}{\longrightarrow} \varepsilon
\end{aligned}
$$

for some $\eta<1$ and $K_{S}<\infty$ (see Lemma 3.12). Since $\varepsilon>0$ is arbitrary we have shown that $\mathbb{E}\left(f\left(R_{n}^{*}\right)\right) \underset{n \rightarrow \infty}{\longrightarrow} \mathbb{E}(f(R))$, and Theorem 1.7 follows.

\section{Application of Kesten's renewal theorem}

In order to prove Theorem 1.9, as mentioned in the introduction, we will use Kesten's renewal theorem [17] which allows us to describe the desired tail asymptotic (1.10). 
Before we state Kesten's theorem we have to introduce necessary definitions and to prove a number of auxiliary results. They are contained in the three lemmas of Sect. 4.1 and they will be used later on to check that the assumptions of Kesten's renewal theorem are satisfied in our settings. The material presented in this section is adapted from $[3,6,7,16]$.

\subsection{Some general results}

At first we define the probability space $\Omega=G^{\mathbb{N}}$. $\mathcal{B}$ or $(X)$ stands for the Borel $\sigma$-field of the space $X$. For any sequence $\omega=\left(a_{1}, a_{2}, \ldots\right) \in \Omega$ we write

$$
S_{n}(\omega)=a_{n} \cdots a_{1} \in G, \quad \text { for } n \in \mathbb{N} \text { and } S_{0}(\omega)=\operatorname{Id} \in G
$$

Let $\theta: \Omega \mapsto \Omega$ be the shift on $\Omega$, i.e.

$$
\theta\left(\left(a_{1}, a_{2}, \ldots\right)\right)=\left(a_{2}, a_{3}, \ldots\right), \quad \text { for every } \omega=\left(a_{1}, a_{2}, \ldots\right) \in \Omega
$$

As in Sect. 2 (see (2.4) and (2.7)), for every $n \in \mathbb{N}$, we define the kernel

$$
q_{n}^{s}(x, \omega)=\prod_{k=1}^{n} q_{1}^{s}\left(S_{k-1}(\omega) \cdot x, a_{k}\right), \quad \text { for every } x \in \mathbb{S}^{+} \text {and } \omega=\left(a_{1}, a_{2}, \ldots\right) \in \Omega
$$

The cocycle property gives a very useful relation, i.e. for every $m, n \in \mathbb{N}, x \in \mathbb{S}^{+}$and $\omega \in \Omega$ we have

$$
q_{m+n}^{s}(x, \omega)=q_{n}^{s}\left(x, S_{n}(\omega)\right) q_{m}^{s}\left(S_{n}(\omega) \cdot x, S_{m}\left(\theta^{n}(\omega)\right)\right) .
$$

The Kolmogorov's consistency theorem guarantees the existence of the probability measure $\mathbb{Q}_{x}^{s}$ on $\Omega$ being the unique extension of measures $q_{k}^{s}(x, a) \mu^{* k}(d a)$. Next we define the probability measure

$$
\mathbb{Q}^{s}=\int_{\mathbb{S}^{+}} \mathbb{Q}_{x}^{s} \pi^{s}(d x), \quad \text { on } \Omega
$$

where $\pi^{s}$ is the unique $Q^{s}$ stationary measure on $\mathbb{S}^{+}$(see Theorem 2.3). By $\mathbb{E}_{x}^{s}$ we denote the expectation corresponding to $\mathbb{Q}_{x}^{s}$. We extend the probability space $\Omega$ to ${ }^{a} \Omega=\mathbb{S}^{+} \times \Omega$. Let ${ }^{a} \theta:{ }^{a} \Omega \mapsto^{a} \Omega$ be the shift defined by

$$
{ }^{a} \theta(x, \omega)=\left(a_{1} \cdot x, \theta(\omega)\right), \quad \text { for every } x \in \mathbb{S}^{+} \text {and } \omega=\left(a_{1}, a_{2}, \ldots\right) \in \Omega \text {. }
$$

We now define the probability measure ${ }^{a} Q^{s}$ on ${ }^{a} \Omega$ as follows

$$
{ }^{a} Q^{s}=\int_{\mathbb{S}^{+}} \delta_{x} \otimes \mathbb{Q}_{x}^{s} \pi^{s}(d x)
$$


In the same way, starting with $\mu_{*}$ instead of $\mu$, and with kernels $q_{n}^{s, *}$ instead of $q_{n}^{s}$ (see (2.7) for the definition) we introduce the measure $\mathbb{Q}_{x}^{s, *}$, and $\mathbb{E}_{x}^{s, *}$ denotes its expectation. Moreover, the probabilities $\mathbb{Q}^{s, *}$ and ${ }^{a} Q^{s, *}$ are defined similarly, i.e.

$$
\mathbb{Q}^{s, *}=\int_{\mathbb{S}^{+}} \mathbb{Q}_{x}^{s, *} \pi_{*}^{s}(d x), \quad \text { and } \quad{ }^{a} Q^{s, *}=\int_{\mathbb{S}^{+}} \delta_{x} \otimes \mathbb{Q}_{x}^{s, *} \pi_{*}^{s}(d x),
$$

where $\pi_{*}^{s}$ is the unique $Q_{*}^{s}$ stationary measure on $\mathbb{S}^{+}$(see Theorem 2.3). Let $\omega^{*}=$ $\left(a_{1}^{*}, a_{2}^{*}, \ldots\right) \in \Omega$ for every $\omega=\left(a_{1}, a_{2}, \ldots\right) \in \Omega$. Then $S_{n}\left(\omega^{*}\right)=a_{n}^{*} \cdots \cdots a_{1}^{*} \in G$.

Remark 4.3 The properties of the stationary measures $\pi^{s}$ and $\pi_{*}^{s}$ developed in Sect. 2 imply that $\left(\Omega, \mathcal{B}\right.$ or $\left.(\Omega), \mathbb{Q}^{s}, \theta\right),\left(\Omega, \mathcal{B}\right.$ or $\left.(\Omega), \mathbb{Q}^{s, *}, \theta\right),\left({ }^{a} \Omega, \mathcal{B}\right.$ or $\left.\left({ }^{a} \Omega\right),{ }^{a} Q^{s},{ }^{a} \theta\right)$ and $\left({ }^{a} \Omega, \mathcal{B}\right.$ or $\left.\left({ }^{a} \Omega\right),{ }^{a} Q^{s, *},{ }^{a} \theta\right)$ are ergodic.

From now we will work with the measures $\mathbb{Q}_{x}^{s, *}, \pi_{*}^{s}, \mathbb{Q}^{s, *}$ and ${ }^{a} Q^{s, *}$. Clearly, all the results stated below remain valid for the measures $\mathbb{Q}_{x}^{s}, \pi^{s}, \mathbb{Q}^{s}$ and ${ }^{a} Q^{s}$.

We begin with the following

Lemma 4.4 Assume that $\mu \in M^{1}(G), s \in I_{\mu}$ and $\Gamma=[$ supp $\mu]$ satisfies condition $(i-p)$. Then there exists $c>0$ such that $\mathbb{Q}_{x}^{s, *} \leq c \mathbb{Q}^{s, *}$ for every $x \in \mathbb{S}^{+}$. Moreover the constant $c$ does not depend on $x \in \mathbb{S}^{+}$.

Proof We can repeat the argument from Sect. 3 in [3].

Lemma 4.5 Assume that $\mu \in M^{1}(G), s \in I_{\mu}$ and $\Gamma=[$ supp $\mu]$ satisfies condition $(i-p)$. Then for every $x \in \mathbb{S}^{+}$we have

$$
\begin{aligned}
& \mathbb{Q}_{x}^{s, *}\left(\left\{\omega \in \Omega: \exists C>0 \forall n \in \mathbb{N} \quad\left|S_{n}(\omega) x\right| \geq C\left\|S_{n}(\omega)\right\|\right\}\right)=1, \quad \text { and } \\
& \mathbb{Q}^{s, *}\left(\left\{\omega \in \Omega: \exists C>0 \forall n \in \mathbb{N} \quad\left|S_{n}(\omega) x\right| \geq C\left\|S_{n}(\omega)\right\|\right\}\right)=1 .
\end{aligned}
$$

Proof Observe that (4.7) implies (4.6). Indeed, let

$$
Z_{x}=\left\{\omega \in \Omega: \exists C>0 \forall n \in \mathbb{N} \quad\left|S_{n}(\omega) x\right| \geq C\left\|S_{n}(\omega)\right\|\right\},
$$

and let $Z_{x}^{c}$ be the complement of $Z_{x}$. Then by Lemma 4.4

$$
\mathbb{Q}_{x}^{s, *}\left(Z_{x}^{c}\right) \leq c \mathbb{Q}^{s, *}\left(Z_{x}^{c}\right)=0
$$

The proof of (4.7) is adapted from [16]. Condition (1.3) yields the existence of $n_{0} \in \mathbb{N}$ and $0<\tau<1$ such that

$$
p=\mathbb{P}^{*}\left(\left\{\omega \in \Omega: S_{n_{0}}(\omega)(i, j)>\tau, \text { for all } 1 \leq i, j \leq d\right\}\right)>0
$$

where $\mathbb{P}^{*}=\mu_{*}^{\otimes \mathbb{N}}$. Let us introduce

$$
T(\omega)=\min \left\{n \geq n_{0}: S_{n_{0}}\left(\theta^{n-n_{0}}(\omega)\right) \in G^{\circ}\right\}
$$


First of all we need to show that

$$
\begin{aligned}
& \mathbb{Q}_{x}^{s, *}(\{\omega \in \Omega: T(\omega)<\infty\})=1, \quad \text { for every } x \in \mathbb{S}^{+} \text {and } \\
& \mathbb{Q}^{s, *}(\{\omega \in \Omega: T(\omega)<\infty\})=1 .
\end{aligned}
$$

Notice that (4.9) immediately gives (4.10), since the event $\{T<\infty\}$ does not depend on $x \in \mathbb{S}^{+}$and $\mathbb{Q}^{s, *}=\int_{\mathbb{S}^{+}} \mathbb{Q}_{x}^{s, *} \pi_{*}^{s}(d x)$.

Assume for a moment that (4.10) holds and prove (4.7). If $x=\left(x_{1}, \ldots, x_{n}\right) \in \mathbb{S}^{+}$ is such that $x>0$ then for any $a \in G$ we have

$$
\begin{aligned}
|a x| \geq & d^{-1 / 2} \sum_{i=1}^{d}\left(a(i, 1) x_{1}+\cdots+a(i, n) x_{n}\right) \geq d^{-1 / 2} \min _{1 \leq i \leq d} x_{i} \sum_{i, j=1}^{d} a(i, j) \\
& \geq d^{-1 / 2} \min _{1 \leq i \leq d} x_{i} \cdot \sup _{|y|=1}\left(\sum_{i=1}^{d}\left(a(i, 1) y_{1}+\cdots+a(i, n) y_{n}\right)^{2}\right)^{1 / 2} \\
& =d^{-1 / 2} \min _{1 \leq i \leq d} x_{i}\|a\| .
\end{aligned}
$$

We will use this inequality to show that (4.7) holds. Now fix an arbitrary $x \in \mathbb{S}^{+}$ and let $\Omega_{1}=\{T<\infty\} \subseteq \Omega$. By assumption, $\mathbb{Q}^{s, *}\left(\Omega_{1}\right)=1$. It is easy to see that $S_{T}\left(\omega^{*}\right) x>0$ for $\omega^{*} \in \Omega_{1}$. Fix $\omega^{*} \in \Omega_{1}$ then for any $n \geq T\left(\omega^{*}\right)$ we have

$$
\begin{aligned}
\left|S_{n}\left(\omega^{*}\right) x\right|=\left|S_{n-T}\left(\theta^{T}\left(\omega^{*}\right)\right) S_{T}\left(\omega^{*}\right) x\right| & \geq d^{-1 / 2} \min _{1 \leq i \leq d}\left(S_{T}\left(\omega^{*}\right) x\right)_{i}\left\|S_{n-T}\left(\theta^{T}\left(\omega^{*}\right)\right)\right\| \\
& \geq d^{-1 / 2} \frac{\min _{1 \leq i \leq d}\left(S_{T}\left(\omega^{*}\right) x\right)_{i}}{\left\|S_{T}\left(\omega^{*}\right)\right\|}\left\|S_{n}\left(\omega^{*}\right)\right\| .
\end{aligned}
$$

It implies that $\left|S_{n}\left(\omega^{*}\right) x\right| \geq C_{T, x}\left(\omega^{*}\right)\left\|S_{n}\left(\omega^{*}\right)\right\|$ holds with the constant $C_{T, x}\left(\omega^{*}\right)>0$ independent of $n \geq T\left(\omega^{*}\right)$, for every $\omega^{*} \in \Omega_{1}$. Recall that $G$ is the multiplicative semigroup of $d \times d$ invertible matrices with positive entries such that every row and every column contains a strictly positive element. Now take $n \leq T\left(\omega^{*}\right)$ and notice that $C_{n, x}\left(\omega^{*}\right)=\frac{\left|S_{n}\left(\omega^{*}\right) x\right|}{\left\|S_{n}\left(\omega^{*}\right)\right\|}>0$, for every $\omega^{*} \in \Omega_{1}$ by the definition of $G$ and $x \in \mathbb{S}^{+}$. Therefore, we take $C\left(\omega^{*}\right)=\min \left\{C_{1, x}\left(\omega^{*}\right), \ldots, C_{T, x}\left(\omega^{*}\right)\right\}>0$, and (4.7) follows.

We need only to prove (4.9). In this purpose we define the events

$$
E_{k}=\left\{\omega \in \Omega: S_{n_{0}}\left(\theta^{k}(\omega)\right)(i, j) \geq \tau, \text { for all } 1 \leq i, j \leq d\right\}, \quad k \in \mathbb{N}
$$

We show that there exists $\gamma \in[0,1)$ such that for all $l \in \mathbb{N}$

$$
\mathbb{Q}_{x}^{s, *}\left(\left\{T>l n_{0}\right\}\right) \leq \mathbb{Q}_{x}^{s, *}\left(\left\{E_{j n_{0}} \text { does not occur for any } 0 \leq j<l\right\}\right) \leq \gamma^{l}
$$


Then (4.11) with Borel-Cantelli lemma yield $\mathbb{Q}_{x}^{S, *}(\{T<\infty\})=1$. In fact it is enough to show that

$$
\begin{aligned}
& \mathbb{Q}_{x}^{s, *}\left(E_{0}^{c} \cap \cdots \cap E_{(l-1) n_{0}}^{c}\right) \leq \gamma \mathbb{Q}_{x}^{s, *}\left(E_{0}^{c} \cap \cdots \cap E_{(l-2) n_{0}}^{c}\right) \leq \gamma^{2} \mathbb{Q}_{x}^{s, *}\left(E_{0}^{c} \cap \cdots \cap E_{(l-3) n_{0}}^{c}\right) \\
& \quad \leq \cdots \text { and inductively } \cdots \leq \gamma^{l} . \\
& \text { Let } r_{s}=\frac{\inf _{x \in \mathbb{S}^{+}} e_{*}^{s}(x)}{\sup _{x \in \mathbb{S}^{+}} e_{*}^{s}(x)} \text {. Then } \\
& \mathbb{Q}_{x}^{s, *}\left(E_{0}^{c} \cap \cdots \cap E_{(l-2) n_{0}}^{c} \cap E_{(l-1) n_{0}}\right) \\
& =\int_{\Omega} \mathbf{1}_{E_{0}^{c} \cap \cdots \cap E_{(l-2) n_{0}}^{c} \cap E_{(l-1) n_{0}}\left(\omega^{*}\right) q_{l n_{0}}^{s, *}\left(x, S_{l n_{0}}\left(\omega^{*}\right)\right) \mu^{* l n_{0}}(d \omega)} \\
& \geq \frac{r_{s} \tau^{s}}{d^{s / 2} \kappa^{n_{0}(s)}} \int_{\Omega} \mathbf{1}_{E_{0}^{c} \cap \cdots \cap E_{(l-2) n_{0}}^{c}\left(\omega^{*}\right) \mathbf{1}_{E_{(l-1) n_{0}}}\left(\omega^{*}\right) q_{(l-1) n_{0}}^{s, *}\left(x, S_{(l-1) n_{0}}\left(\omega^{*}\right)\right) \mu^{*} \ln n_{0}(d \omega)} \\
& =\frac{r_{s} \tau^{s}}{d^{s / 2} \kappa^{n_{0}(s)}} \mathbb{P}^{*}\left(E_{(l-1) n_{0}}\right) \mathbb{Q}_{x}^{s, *}\left(E_{0}^{c} \cap \cdots \cap E_{(l-2) n_{0}}^{c}\right) \\
& =\frac{p r_{s} \tau^{s}}{d^{s / 2} \kappa^{n_{0}(s)}} \mathbb{Q}_{x}^{s, *}\left(E_{0}^{c} \cap \cdots \cap E_{(l-2) n_{0}}^{c}\right),
\end{aligned}
$$

( $n_{0} \in \mathbb{N}$ and $\tau>0$ were defined in (4.8)) since by (4.2) we have the following lower bound

$$
\begin{aligned}
& \mathbf{1}_{E_{(l-1) n_{0}}}\left(\omega^{*}\right) q_{l n_{0}}^{s, *}\left(x, S_{l n_{0}}\left(\omega^{*}\right)\right) \\
& =\mathbf{1}_{E_{(l-1) n_{0}}}\left(\omega^{*}\right) q_{(l-1) n_{0}}^{s, *}\left(x, S_{(l-1) n_{0}}\left(\omega^{*}\right)\right) q_{n_{0}}^{s, *}\left(S_{(l-1) n_{0}}\left(\omega^{*}\right) \cdot x, S_{n_{0}}\left(\theta^{(l-1) n_{0}}\left(\omega^{*}\right)\right)\right) \\
& \geq \frac{r_{s}}{\kappa^{n_{0}(s)}} \mathbf{1}_{E_{(l-1) n_{0}}}\left(\omega^{*}\right) q_{(l-1) n_{0}}^{s, *}\left(x, S_{(l-1) n_{0}}\left(\omega^{*}\right)\right)\left|S_{n_{0}}\left(\theta^{(l-1) n_{0}}\left(\omega^{*}\right)\right)\left(S_{(l-1) n_{0}}\left(\omega^{*}\right) \cdot x\right)\right|^{s} \\
& \geq \frac{r_{s}}{d^{s / 2} \kappa^{n_{0}(s)}} \mathbf{1}_{E_{(l-1) n_{0}}}\left(\omega^{*}\right) q_{(l-1) n_{0}}^{s, *}\left(x, S_{(l-1) n_{0}}\left(\omega^{*}\right)\right) \\
& \times\left(\sum_{i=1}^{d} S_{n_{0}}\left(\theta^{(l-1) n_{0}}\left(\omega^{*}\right)\right)\left(S_{(l-1) n_{0}}\left(\omega^{*}\right) \cdot x\right)_{i}\right)^{s} \\
& \geq \frac{r_{s} \tau^{s}}{d^{s / 2} \kappa^{n_{0}(s)}} \mathbf{1}_{E_{(l-1) n_{0}}}\left(\omega^{*}\right) q_{(l-1) n_{0}}^{s, *}\left(x, S_{(l-1) n_{0}}\left(\omega^{*}\right)\right) .
\end{aligned}
$$

Let $0<\gamma_{s}=\min \left\{1, \frac{p r_{s} \tau^{s}}{d^{s / 2} \kappa^{n} 0(s)}\right\}$. For $\gamma=1-\gamma_{s} \in[0,1)$, by (4.13), we obtain that

$$
\begin{aligned}
\mathbb{Q}_{x}^{s, *}\left(E_{0}^{c} \cap \cdots \cap E_{(l-2) n_{0}}^{c} \cap E_{(l-1) n_{0}}^{c}\right) & \leq \gamma \mathbb{Q}_{x}^{s, *}\left(E_{0}^{c} \cap \cdots \cap E_{(l-2) n_{0}}^{c} \cap G_{(l-1) n_{0}}\right) \\
& =\gamma \mathbb{Q}_{x}^{s, *}\left(E_{0}^{c} \cap \cdots \cap E_{(l-2) n_{0}}^{c}\right) .
\end{aligned}
$$

This finishes the proof of (4.12) and completes the proof of the lemma. 
Lemma 4.14 Assume that $\mu \in M^{1}(G), s \in I_{\mu}$ and $\Gamma=[$ supp $\mu]$ satisfies condition $(i-p)$. Assume additionally that $\int_{G}\|a\|^{s} \log ^{+}\|a\| \mu(d a)<\infty$. Then for any $x \in \mathbb{S}^{+}$

$$
\lim _{n \rightarrow \infty} \frac{1}{n} \log \left|S_{n}(\omega) x\right|=\lim _{n \rightarrow \infty} \frac{1}{n} \log \left\|S_{n}(\omega)\right\|=\alpha(s), \quad \mathbb{Q}_{x}^{s, *} \text { and } \mathbb{Q}^{s, *} \text { a.s. }
$$

where

$$
\alpha(s)=\int_{\mathbb{S}^{+}} \int_{G} \log |a x| q_{1}^{s, *}(x, a) \mu_{*}(d a) \pi_{*}^{s}(d x) .
$$

Proof We show that $f(x, \omega)=\log \left|S_{1}(\omega) x\right|$ is ${ }^{a} Q^{s, *}$ integrable. Observe that there exists $0<\delta<1$ such that

$$
0<|a x|<\delta \Longrightarrow|a x|^{s} \log |a x|^{-1} \leq 1
$$

Then

$$
\begin{aligned}
{ }^{a} Q^{s, *}(|f|)= & \int_{\mathbb{S}^{+}} \int_{\Omega}|\log | S_{1}(\omega) y \| \delta_{x}(d y) \mathbb{Q}_{x}^{s, *}(d \omega) \pi_{*}^{s}(d x) \\
= & \int_{\mathbb{S}^{+}} \int_{G}|a x|^{s}|\log | a x \| \frac{e_{*}^{s}(a \cdot x)}{\kappa(s) e_{*}^{s}(x)} \mu_{*}(d a) \pi_{*}^{s}(d x) \\
\leq & C_{S} \int_{\mathbb{S}^{+}} \int_{G}|a x|^{s}|\log | a x \| \mu_{*}(d a) \pi_{*}^{s}(d x) \\
\leq & C_{S} \int_{G}\|a\|^{s} \log ^{+}\|a\| \mu(d a)+C_{S} \int_{\mathbb{S}^{+}} \int_{G}|a x|^{s} \log ^{-}|a x| \mu_{*}(d a) \pi_{*}^{s}(d x) \\
\leq & C_{S} \int_{G}\|a\|^{s} \log ^{+}\|a\| \mu(d a)+C_{s} \mu_{*} \otimes \pi_{*}^{s}\left(\left\{(a, x) \in G \times \mathbb{S}^{+}: 0<|a x|<\delta\right\}\right) \\
& +C_{S} \log \left(\frac{1}{\delta}\right) \mu_{*} \otimes \pi_{*}^{s}\left(\left\{(a, x) \in G \times \mathbb{S}^{+}: \delta<|a x| \leq 1\right\}\right) \\
\leq & C_{s} \int_{G}\|a\|^{s} \log ^{+}\|a\| \mu(d a)+C_{s}\left(1+\log \left(\frac{1}{\delta}\right)\right)<\infty .
\end{aligned}
$$

Hence in view of Remark 4.3, on the one hand, by the Birkhoff ergodic theorem (applied to ${ }^{a} Q^{s, *}$ and ${ }^{a} \theta$ ) we obtain

$$
\begin{aligned}
& { }^{a} Q^{s, *}\left(\left\{(x, \omega) \in{ }^{a} \Omega: \lim _{n \rightarrow \infty} \frac{1}{n} \log \left|S_{n}(\omega) x\right|\right.\right. \\
& \left.\left.\quad=\lim _{n \rightarrow \infty} \frac{1}{n} \cdot \sum_{k=0}^{n-1} f \circ^{a} \theta^{k}(x, \omega)={ }^{a} Q^{s, *}(f)=\alpha(s)\right\}\right)=1
\end{aligned}
$$


On the other hand by the Kingman subadditive ergodic theorem (applied to $Q^{s, *}$ and $\theta$ ) we have that for every $s \in I_{\mu}$ there exists $\alpha_{s} \in \mathbb{R}$ such that

$$
\mathbb{Q}^{s, *}\left(\left\{\omega \in \Omega: \lim _{n \rightarrow \infty} \frac{1}{n} \log \left\|S_{n}(\omega)\right\|=\alpha_{s}\right\}\right)=1 .
$$

Define $\Omega^{\prime}=\left\{\omega \in \Omega: \exists C>0 \forall n \in \mathbb{N}\left|s_{n}(\omega) x\right| \geq C\left\|S_{n}(\omega)\right\|\right.$ and $\lim _{n \rightarrow \infty} \frac{1}{n} \log$ $\left.\left\|S_{n}(\omega)\right\|=\alpha_{s}\right\}$, for every $x \in \mathbb{S}^{+}$. By Lemma 4.5 and calculations stated above we know that $\mathbb{Q}^{s, *}\left(\Omega^{\prime}\right)=1$. Fix arbitrary $x \in \mathbb{S}^{+}$, take any $\omega^{*} \in \Omega^{\prime}$ and notice that

$$
0<C_{x}\left(\omega^{*}\right) \leq \frac{\left|S_{n}\left(\omega^{*}\right) x\right|}{\left\|S_{n}\left(\omega^{*}\right)\right\|} \leq 1
$$

imply

$$
\begin{aligned}
& \frac{1}{n} \log C_{x}\left(\omega^{*}\right)+\frac{1}{n} \log \left\|S_{n}\left(\omega^{*}\right)\right\| \leq \frac{1}{n} \log \frac{\left|S_{n}\left(\omega^{*}\right) x\right|}{\left\|S_{n}\left(\omega^{*}\right)\right\|}+\frac{1}{n} \log \left\|S_{n}\left(\omega^{*}\right)\right\| \\
& \leq \frac{1}{n} \log \left\|S_{n}\left(\omega^{*}\right)\right\| .
\end{aligned}
$$

Since $\lim _{n \rightarrow \infty} \frac{1}{n} \log C_{x}\left(\omega^{*}\right)=0$ we have

$$
\mathbb{Q}^{s, *}\left(\left\{\omega \in \Omega: \lim _{n \rightarrow \infty} \frac{1}{n} \log \left|S_{n}(\omega) x\right|=\alpha_{s}\right\}\right)=1 .
$$

And so, in view of Lemma 4.4,

$$
\mathbb{Q}_{x}^{s, *}\left(\left\{\omega \in \Omega: \lim _{n \rightarrow \infty} \frac{1}{n} \log \left|S_{n}(\omega) x\right|=\alpha_{s}\right\}\right)=1,
$$

for all $x \in \mathbb{S}^{+}$(by considering complements). Since ${ }^{a} Q^{s, *}=\int_{\mathbb{S}^{+}} \delta_{x} \otimes \mathbb{Q}_{x}^{s, *} \pi_{*}^{s}(d x)$ we get $\alpha(s)=\alpha_{s}$ and Lemma 4.14 follows.

\subsection{Kesten's renewal theorem}

For $x \in \mathbb{S}^{+}$and $\omega \in \Omega$ define $X_{0}(\omega)=x$, and for $n \in \mathbb{N}$

$$
X_{n}(\omega)=g_{n}(\omega) \cdot X_{n-1}(\omega)=S_{n}(\omega) \cdot x
$$

and

$$
V_{n}(\omega)=\log \left|S_{n}(\omega) x\right|=\sum_{i=1}^{n} U_{i}(\omega), \quad \text { where } \quad U_{i}(\omega)=\log \left|g_{i}(\omega) X_{i-1}(\omega)\right|
$$


Let $F(d t \mid x, y)$ be the conditional law of $U_{1}$, given $X_{0}=x, X_{1}=y$, i.e.

$$
\mathbb{Q}_{x}^{s, *}\left(X_{1} \in A, U_{1} \in B\right)=\int_{A} \int_{B} F(d t \mid x, y) Q_{*}^{s}(x, d y) .
$$

A function $g: \mathbb{S}^{+} \times \mathbb{R} \rightarrow \mathbb{R}$ is called direct Riemann integrable $(d \mathcal{R} i)$, if it is $\operatorname{Bor}\left(\mathbb{S}^{+}\right) \times \operatorname{Bor}(\mathbb{R})$ measurable and for every fixed $x \in \mathbb{S}^{+}$and $0<L<\infty$ the function $t \mapsto g(x, t)$ is Riemann integrable on $[-L, L]$, and satisfies

$$
\sum_{k=0}^{\infty} \sum_{l=-\infty}^{\infty}(k+1) \sup \left\{|g(x, t)|: x \in C_{k+1} \backslash C_{k}, \quad \text { and } \quad t \in[l, l+1]\right\}<\infty,
$$

where

$$
C_{k}=\left\{x \in \mathbb{S}^{+}: \mathbb{Q}_{x}^{s, *}\left(\left\{\frac{V_{m}}{m} \geq \frac{1}{k}, \text { for all } m \geq k\right\}\right) \geq \frac{1}{2}\right\}, \quad \text { for all } k \in \mathbb{N} \text {. }
$$

For the reader's convenience we formulate Kesten's renewal theorem [17].

Theorem 4.19 Assume the following conditions are satisfied:

- Condition I.1 There exists $\pi_{*}^{s} \in M^{1}\left(\mathbb{S}^{+}\right)$such that $\pi_{*}^{s} Q_{*}^{s}=\pi_{*}^{s}$ and for every open set $U \subseteq \mathbb{S}^{+}$with $\pi_{*}^{s}(U)>0, \mathbb{Q}_{x}^{s, *}\left(X_{n} \in U\right.$ for some $\left.n \in \mathbb{N}\right)=1$ for every $x \in \mathbb{S}^{+}$.

\section{- Condition I.2}

$$
\iint_{\mathbb{S}^{+}} \int_{\mathbb{R}}|t| F(d t \mid x, y) Q_{*}^{s}(x, d y) \pi_{*}^{s}(d x)<\infty
$$

and for all $x \in \mathbb{S}^{+}$,

$$
\lim _{n \rightarrow \infty} \frac{V_{n}}{n}=\alpha(s)=\int t F(d t \mid x, y) Q_{*}^{s}(x, d y) \pi_{*}^{s}(d x)>0 \quad \mathbb{Q}_{x}^{s, *}-\text { a.e. }
$$

- Condition I.3 There exists a sequence $\left\{\zeta_{i}\right\} \subset \mathbb{R}$ such that the group generated by $\zeta_{i}$ is dense in $\mathbb{R}$ and such that for each $\zeta_{i}$ and $\lambda>0$ there exists $y=y\left(\zeta_{i}, \lambda\right) \in \mathbb{S}^{+}$ with the following property: for each $\varepsilon>0$, there exists $A \in \mathcal{B}$ or $\left(\mathbb{S}^{+}\right)$with $\pi_{*}^{s}(A)>0$ and $m_{1}, m_{2} \in \mathbb{N}, \tau \in \mathbb{R}$ such that for any $x \in A$

$$
\begin{gathered}
\mathbb{Q}_{x}^{s, *}\left\{\left|X_{m_{1}}-y\right|<\varepsilon,\left|V_{m_{1}}-\tau\right| \leq \lambda\right\}>0, \\
\mathbb{Q}_{x}^{s, *}\left\{\left|X_{m_{2}}-y\right|<\varepsilon,\left|V_{m_{2}}-\tau-\zeta_{i}\right| \leq \lambda\right\}>0 .
\end{gathered}
$$


- Condition I.4 For each fixed $x \in \mathbb{S}^{+}, \varepsilon>0$ there exists $r_{0}=r_{0}(x, \varepsilon)>0$ such that for all real valued functions $f$ measurable with respect to $\mathcal{B o r}\left(\left(\mathbb{S}^{+} \times \mathbb{R}\right)^{\mathbb{N}}\right)$ and for all $y \in \mathbb{S}^{+}$with $|x-y|<r_{0}$ one has:

$$
\begin{aligned}
& \mathbb{E}_{x}^{s, *} f\left(X_{0}, V_{0}, X_{1}, V_{1}, \ldots\right) \leq \mathbb{E}_{y}^{s, *} f^{\varepsilon}\left(X_{0}, V_{0}, X_{1}, V_{1}, \ldots\right)+\varepsilon|f|_{\infty}, \\
& \mathbb{E}_{y}^{s, *} f\left(X_{0}, V_{0}, X_{1}, V_{1}, \ldots\right) \leq \mathbb{E}_{x}^{s, *} f^{\varepsilon}\left(X_{0}, V_{0}, X_{1}, V_{1}, \ldots\right)+\varepsilon|f|_{\infty},
\end{aligned}
$$

where $f^{\varepsilon}\left(x_{0}, v_{0}, x_{1}, v_{1}, \ldots\right)=\sup \left\{f\left(y_{0}, u_{0}, y_{1}, u_{1}, \ldots\right): \forall i \in \mathbb{N}\left|x_{i}-y_{i}\right|+\right.$ $\left.\left|v_{i}-u_{i}\right|<\varepsilon\right\}$.

If a function $g: \mathbb{S}^{+} \times \mathbb{R} \mapsto \mathbb{R}$ is jointly continuous and $(d \mathcal{R} i)$, then for every $x \in \mathbb{S}^{+}$

$$
\lim _{t \rightarrow \infty} \mathbb{E}_{x}^{s, *}\left(\sum_{n=0}^{\infty} g\left(X_{n}, t-V_{n}\right)\right)=\frac{1}{\alpha(s)} \int_{\mathbb{S}^{+}}\left(\int_{\mathbb{R}} g(y, x) d x\right) \pi_{*}^{s}(d y),
$$

for $\alpha(s)$ defined in (4.20).

In the next four subsections we indicate how the material developed in Sects. 2 and 4.1, under the hypotheses of Theorem 1.9, may be used to check the assumptions of Theorem 4.19. From now we will work with the measures $\mathbb{Q}_{x}^{\chi, *}$ for $x \in \mathbb{S}^{+}$, where $\chi>0$ solves equation $\kappa(\chi)=\frac{1}{N}$. Such $\chi>0$ exists since $\kappa(s)$ is strictly log-convex and $\lim _{s \rightarrow s_{\infty}} \kappa(s)>\frac{1}{N}$, (see Theorems 1.9 and 2.3). We are going to prove that Conditions I.1-I.4 are satisfied for $s=\chi$.

\subsection{Condition I.1.}

Proof of Condition I.1. Theorem 2.3 with Breiman's strong law of large numbers [2] allow us to repeat the argument contained in Sect. 5 in [3].

\subsection{Condition I.2.}

Proof of Condition I.2. We know that $\int_{G}\|a\|^{\chi} \log ^{+}\|a\| \mu(d a)<\infty$, hence

$$
\begin{aligned}
\iint_{\mathbb{S}^{+}} & \int_{\mathbb{S}^{+}}|t| F(d t \mid x, y) Q_{*}^{\chi}(x, d y) \pi_{*}^{\chi}(d x) \\
& =\int_{\mathbb{S}^{+}} \int_{\Omega}|\log | a x|| q_{1}^{\chi, *}(x, a) \mu_{*}(d a) \pi_{*}^{\chi}(d x)<\infty,
\end{aligned}
$$

by the arguments of Lemma 4.14 applied to $s=\chi$. The only point remaining concerns the positivity of $\alpha(\chi)$ defined in Lemma 4.14 (see also (4.20)).

Notice that if $\varepsilon>0$ is sufficiently small, then for every $t \in(\chi-\varepsilon, \chi)$, we have $\kappa(t)<\kappa(\chi)$, since $\kappa(s)$ is strictly log-convex and $\lim _{s \rightarrow s_{\infty}} \kappa(s)>\frac{1}{N}$, (see Theorems 1.9 and 2.3). Fix $t \in(\chi-\varepsilon, \chi)$ such that $\chi / t \leq 4 / 3$ and take $\gamma>0$ such that $\kappa(t) e^{\gamma}<\kappa(\chi)$. In view of inequality (3.11), there is $C>0$ such that 


$$
\int_{G}\|a\|^{t} \mu_{*}^{* n}(d a) \leq C \kappa^{n}(t) e^{\gamma n / 3}, \quad \text { for every } n \in \mathbb{N}
$$

since $1 \leq e^{\gamma / 3}$. Fix $x \in \mathbb{S}^{+}$. Then for $\delta=\gamma / 3$ we have

$$
\mu_{*}^{* n}\left(\left\{a \in G:|a x|^{t}>e^{-\delta n}\right\}\right) \leq e^{\delta n} \int_{G}|a x|^{t} \mu_{*}^{* n}(d a) \leq C \kappa^{n}(t) e^{2 \gamma n / 3},
$$

Now let $\rho=\gamma / 6$. Throughout the proof will use the convention that $D>0$ stands for a large positive constant whose value varies from occurrence to occurrence. Then

$$
\begin{aligned}
& \mathbb{Q}_{x}^{\chi, *}\left(\left\{\omega \in \Omega:\left|S_{n}(\omega) x\right|^{t}<e^{\rho n}\right\}\right)=\int_{G} \mathbf{1}_{\left\{a \in G:|a x|^{t}<e^{\rho n}\right\}} q_{n}^{\chi, *}(x, a) \mu_{*}^{* n}(d a) \\
& \leq \frac{D}{\kappa^{n}(\chi)} \int_{G} \mathbf{1}_{\left\{a \in G:|a x|^{t}<e^{\rho n}\right\}}|a x|^{\chi} \mu_{*}^{* n}(d a) \\
& \leq \frac{D}{\kappa^{n}(\chi)} \int_{G} \mathbf{1}_{\left\{a \in G:|a x|^{t}<e^{-\delta n\}}|a x|^{\chi}\right.} \mu_{*}^{* n}(d a)+\frac{D}{\kappa^{n}(\chi)} \\
& \times \int_{G} \mathbf{1}_{\left\{a \in G: e^{-\delta n} \leq|a x|^{t}<e^{\rho n\}}|a x|^{\chi} \mu_{*}^{* n}(d a)\right.} \\
& \leq \frac{D \kappa^{n}(t)}{\kappa^{n}(\chi)} \cdot \frac{1}{\kappa^{n}(t)} e^{-\frac{\chi-t}{t} \delta n} \int_{G}|a x|^{t} \frac{e_{*}^{t}(a \cdot x)}{e_{*}^{t}(x)} \mu_{*}^{* n}(d a) \\
& +\frac{D}{\kappa^{n}(\chi)} \mu_{*}^{* n}\left(\left\{a \in G:|a x|^{t}>e^{-\delta n}\right\}\right) e^{\frac{\rho n \chi}{t}} \\
& \leq D e^{-\left(\gamma+\frac{\chi-t}{t} \delta\right)_{n}}+D e^{-\gamma n} e^{2 \gamma n / 3} e^{\rho n \chi / t} \\
& \leq D e^{-\left(\gamma+\frac{\chi-t}{t} \delta\right) n}+D e^{-\gamma n / 3+2 \gamma n / 9} \leq D e^{-\beta n},
\end{aligned}
$$

for some $\beta>0$. Thus

$$
\sum_{n \in \mathbb{N}} \mathbb{Q}_{x}^{\chi, *}\left(\left\{\omega \in \Omega: \log \left|S_{n}(\omega) x\right|<\frac{\rho n}{t}\right\}\right)<\infty
$$

Therefore, by the Borel-Cantelli lemma we obtain that for every $x \in \mathbb{S}^{+}$

$$
\mathbb{Q}_{x}^{\chi, *}\left(\left\{\omega \in \Omega: \liminf _{n \rightarrow \infty} \frac{\log \left|S_{n}(\omega) x\right|}{n} \geq \frac{\rho}{t}>0\right\}\right)=1 .
$$

This shows that $\alpha(\chi)>0 \mathbb{Q}_{x}^{\chi, *}$ a.s. for every $x \in \mathbb{S}^{+}$and finishes the proof of Condition I.2. 


\subsection{Condition I.3.}

Proof of Condition I.3. Proposition 2.1 and Theorem 2.3 allow us to use arguments from Section 5 in [3].

\subsection{Condition I.4.}

Proof of Condition I.4. The proof is a consequence of Lemma 4.5 and the argument given by Kesten [16].

\subsection{Direct Riemann integrability}

Now we derive an interesting criterium which significantly simplifies condition (4.17).

Lemma 4.23 Assume that the hypotheses of Theorem 1.9 are satisfied. If $h$ is any bounded and continuous function on $\mathbb{S}^{+} \times \mathbb{R}$ which satisfies

$$
\sum_{l=-\infty}^{\infty} \sup \left\{|h(x, t)|: x \in \mathbb{S}^{+}, \text {and } t \in[l, l+1]\right\}<\infty
$$

then $h$ is direct Riemann integrable i.e. it satisfies condition (4.17).

Proof We give only a sketch of the proof, for more details we refer to [3]. First of all we prove that $C_{k}=\mathbb{S}^{+}$, for some sufficiently large $k \in \mathbb{N},\left(C_{k}\right.$ was defined in (4.18)). Then obviously (4.24) implies (4.17). There is a finite number $N_{1}$ of points such that $\mathbb{S}^{+} \subseteq \bigcup_{i=1}^{N_{1}} B\left(x_{i}, 2\right)$, since $\mathbb{S}^{+}$is compact. Let

$$
\begin{aligned}
\Omega^{\prime}= & \left\{\lim _{n \rightarrow \infty} \frac{\log \left|S_{n} x_{i}\right|}{n}=\alpha(\chi)>0, \text { and } \exists C>0 \forall n \in \mathbb{N}\left|S_{n} x_{i}\right| \geq C\left\|S_{n}\right\|,\right. \\
& \text { for all } \left.1 \leq i \leq N_{1}\right\}
\end{aligned}
$$

Then $\mathbb{Q}^{\chi, *}\left(\Omega^{\prime}\right)=1$, by Lemmas 4.5 and 4.14. Take any $y \in \mathbb{S}^{+}$, then there exists $1 \leq i \leq N_{1}$ such that $y \in B\left(x_{i}, 2\right)$. This implies the existence of $m_{0} \in \mathbb{N}$ such that

$$
\mathbb{Q}^{\chi, *}\left(\left\{\omega \in \Omega: \frac{\log \left|S_{n}(\omega) y\right|}{n}>\alpha(\chi) / 2, \text { for all } n \geq m_{0}\right\}\right) \geq 1-\frac{1}{2 c}
$$

with the constant $c>0$ defined in Lemma 4.4. Taking any $1 / k \leq \min \left\{\alpha(s) / 2,1 / m_{0}\right\}$ Lemma 4.23 follows. 


\section{Proof of the main theorem}

In this section we give a detailed proof of Theorem 1.9. For that we consider the following smooth version of $\mathbb{P}(\{\langle R, u\rangle>t\})$

$$
G(u, t)=\frac{1}{e^{t} e_{*}^{\chi}(u)} \int_{0}^{e^{t}} r^{\chi} \mathbb{P}(\{\langle R, u\rangle>r\}) d r, \quad \text { where }(u, t) \in \mathbb{S}^{+} \times \mathbb{R},
$$

where $R \in \mathbb{R}_{+}^{d}$ solves Eq. (1.1). Let $\mathbf{B}\left(\mathbb{S}^{+} \times \mathbb{R}\right)$ be the space of all bounded measurable functions on $\mathbb{S}^{+} \times \mathbb{R}$. Define a linear operator $\Theta: \mathbf{B}\left(\mathbb{S}^{+} \times \mathbb{R}\right) \mapsto \mathbf{B}\left(\mathbb{S}^{+} \times \mathbb{R}\right)$ given by the formula

$$
\begin{aligned}
\Theta f(u, t) & =\mathbb{E}_{u}^{\chi, *}\left(f\left(X_{1}, t-V_{1}\right)\right) \\
& =\frac{1}{\kappa(\chi)} \int_{\Omega} f\left(S_{1}\left(\omega^{*}\right) \cdot u, t-\log \left|S_{1}\left(\omega^{*}\right) u\right|\right) \frac{e_{*}^{\chi}\left(S_{1}\left(\omega^{*}\right) \cdot u\right)}{e_{*}^{\chi}(u)}\left|S_{1}\left(\omega^{*}\right) u\right|^{\chi} \mathbb{P}(d \omega) .
\end{aligned}
$$

Observe that for every $n \in \mathbb{N}$

$$
\Theta^{n} f(u, t)=\mathbb{E}_{u}^{\chi, *}\left(f\left(X_{n}, t-V_{n}\right)\right) .
$$

First we express $G(u, t)$ as a potential of a function $g(u, t)$ that turns out later on to be direct Riemann integrable. Recall that $A, A_{1}, A_{2}, \ldots \in G$ is a sequence of independent copies of $G$-valued random matrix $A$ distributed according to $\mu$ and they are independent of $R$. For $n \in \mathbb{N}$ let $S_{n}=A_{n} \cdots A_{1} \in G$.

Lemma 5.2 Assume that the hypotheses of Theorem 1.9 are satisfied. Let $G(u, t)$ be the function defined in (5.1), and

$$
G_{0}(u, t)=\frac{N}{e^{t} e_{*}^{\chi}(u)} \int_{0}^{e^{t}} r^{\chi} \mathbb{P}(\{\langle A R, u\rangle>r\}) d r
$$

then

$$
\begin{aligned}
& G_{0}(u, t)=\Theta G(u, t), \quad \text { and } \\
& \lim _{n \rightarrow \infty} \Theta^{n} G(u, t)=\lim _{n \rightarrow \infty} \mathbb{E}_{u}^{\chi, *}\left(G\left(X_{n}, t-V_{n}\right)\right)=0 .
\end{aligned}
$$

Moreover,

$$
G(u, t)=\sum_{n=0}^{\infty} \Theta^{n} g(u, t), \quad \text { where }
$$




$$
g(u, t)=\frac{1}{e^{t} e_{*}^{\chi}(u)} \int_{0}^{e^{t}} r^{\chi}(\mathbb{P}(\{\langle R, u\rangle>r\})-N \mathbb{P}(\{\langle A R, u\rangle>r\})) d r .
$$

Proof First of all we show $G_{0}(u, t)=\Theta G(u, t)$. Indeed,

$$
\begin{aligned}
G_{0}(u, t) & =\frac{N}{e^{t} e_{*}^{\chi}(u)} \int_{0}^{e^{t}} r^{\chi} \mathbb{P}\left(\left\{\left\langle R, A^{*} \cdot u\right\rangle\left|A^{*} u\right|>r\right\}\right) d r \\
& =\mathbb{E}\left(\frac{N}{e^{t} e_{*}^{\chi}(u)} \int_{0}^{e^{t}} r^{\chi} \mathbf{1}\left(\frac{r}{\left|A^{*} u\right|}, \infty\right)\left(\left\langle R, A^{*} \cdot u\right\rangle\right) d r\right) \\
& =\mathbb{E}\left(\frac{N}{\frac{e^{t}}{\left|A^{*} u\right|} e_{*}^{\chi}(u)} \int_{0}^{\frac{e^{t}}{\left|A^{*} u\right|}} r^{\chi} \mathbf{1}_{(r, \infty)}\left(\left\langle R, A^{*} \cdot u\right|\right)\left|A^{*} u\right|^{\chi} d r\right) \\
& =\mathbb{E}\left(\frac{1}{\frac{e^{t}}{\left|A^{*} u\right|} e_{*}^{\chi}\left(A^{*} \cdot u\right)} \int_{0}^{\frac{e^{t}}{\left|A^{*} u\right|}} r^{\chi} \mathbf{1}_{(r, \infty)}\left(\left|R, A^{*} \cdot u\right\rangle\right) d r \frac{1}{\kappa(\chi)} \frac{e_{*}^{\chi}\left(A^{*} \cdot u\right)}{e_{*}^{\chi}(u)}\left|A^{*} u\right|^{\chi}\right) \\
& =\Theta G(u, t) .
\end{aligned}
$$

Now we have

$\Theta^{n} G(u, t)$

$$
=\mathbb{E}_{u}^{\chi, *}\left(G\left(X_{n}, t-V_{n}\right)\right)=\mathbb{E}^{*}\left(G\left(S_{n} \cdot u, t-\log \left|S_{n} u\right|\right) \frac{1}{\kappa^{n}(\chi)} \frac{e_{*}^{\chi}\left(S_{n} \cdot u\right)}{e_{*}^{\chi}(u)}\left|S_{n} u\right|^{\chi}\right)
$$$$
=N^{n} \mathbb{E}^{*}\left(\frac{\left|S_{n} u\right|}{e^{t} e_{*}^{\chi}\left(S_{n} \cdot u\right)} \int_{0}^{\frac{e^{t}}{\left|S_{n} u\right|}} r^{\chi} \mathbf{1}_{(r, \infty)}\left(\left\langle R, S_{n} \cdot u\right\rangle\right) \frac{e_{*}^{\chi}\left(S_{n} \cdot u\right)}{e_{*}^{\chi}(u)}\left|S_{n} u\right|^{\chi} d r\right)
$$$$
=N^{n} \mathbb{E}^{*}\left(\frac{\left|S_{n} u\right|^{\chi+1}}{e^{t} e_{*}^{\chi}(u)} \int_{0}^{\substack{e^{t} \\\left|S_{n} u\right|}} r^{\chi} \mathbf{1}_{(r, \infty)}\left(\left\langle R, S_{n} \cdot u\right\rangle\right) d r\right)
$$

$=N^{n} \mathbb{E}^{*}\left(\frac{\left|S_{n} u\right|^{\chi+1}}{e^{t} e_{*}^{\chi}(u)} \int_{0}^{\frac{e^{t}}{\left|S_{n} u\right|}} r^{\chi} \mathbf{1}_{\left(\left|S_{n} u\right| r, \infty\right)}\left(\left|S_{n}^{*} R, u\right\rangle\right) d r\right)$ 


$$
\begin{aligned}
& =\frac{N^{n}}{e^{t} e_{*}^{\chi}(u)} \int_{0}^{e^{t}} r^{\chi} \mathbb{E}^{*}\left(\mathbf{1}_{(r, \infty)}\left(\left\langle S_{n}^{*} R, u\right\rangle\right) d r\right) \\
& =\frac{N^{n}}{e^{t} e_{*}^{\chi}(u)} \int_{0}^{e^{t}} r^{\chi} \mathbb{E}\left(\mathbf{1}_{(r, \infty)}\left(\left\langle A_{1} \cdots A_{n} R, u\right\rangle\right) d r\right),
\end{aligned}
$$

where $S_{n}=A_{n} \cdots A_{1}$. By the continuity of $I_{\mu} \ni s \mapsto \kappa(s)$ (see Theorem 2.3) we can find $p<\chi$, such that $\kappa(p)=\frac{1-\varepsilon}{N}$, for some $\varepsilon>0$, then

$\mathbb{E}\left(\mathbf{1}_{(r, \infty)}\left(\left\langle A_{1} \cdots A_{n} R, u\right\rangle\right)\right) \leq \frac{\mathbb{E}\left(\left\|A_{1} \cdots A_{n}\right\|^{p}\right) \mathbb{E}\left(|R|^{p}\right)}{r^{p}} \leq \frac{C \kappa^{n}(p) \mathbb{E}\left(|R|^{p}\right)}{r^{p}}$

This implies that

$$
\begin{aligned}
\Theta^{n} G(u, t) & =\frac{N^{n}}{e^{t} e_{*}^{\chi}(u)} \int_{0}^{e^{t}} r^{\chi} \mathbb{E}\left(\mathbf{1}_{(r, \infty)}\left(\left\langle A_{1} \cdots A_{n} R, u\right\rangle\right) d r\right) \\
& \leq \frac{C N^{n}}{e^{t} e_{*}^{\chi}(u)} \int_{0}^{e^{t}} r^{\chi-p} \kappa^{n}(p) \mathbb{E}\left(|R|^{p}\right) d r \\
& \leq \frac{C N^{n}}{e^{t} e_{*}^{\chi}(u)} \mathbb{E}\left(|R|^{p}\right)\left(\frac{1-\varepsilon}{N}\right)^{n} \int_{0}^{e^{t}} r^{\chi-p} d r \\
& \leq \frac{C \mathbb{E}\left(|R|^{p}\right)}{e_{*}^{\chi}(u)} e^{t(\chi-p)}(1-\varepsilon)^{n} \underset{n \rightarrow \infty}{\longrightarrow} 0 .
\end{aligned}
$$

Now it is easy to see that for any $n \in \mathbb{N}$ we have

$$
G(u, t)=g(u, t)+\Theta g(u, t)+\Theta^{2} g(u, t)+\cdots+\Theta^{n-1} g(u, t)+\Theta^{n} G(u, t),
$$

and (5.5) follows. This completes the proof of Lemma 5.4.

Lemmas 5.8 and 5.16 below imply that $g(u, t)$ is direct Riemann integrable. Lemmas 5.7, 5.12 and 5.14 contain some necessary technicalities.

Lemma 5.7 Assume that the hypotheses of Theorem 1.9 are satisfied. Then $\mathbb{P}(\{\langle R, u\rangle=r\})=0$, for every $(u, r) \in \mathbb{S}^{+} \times \mathbb{R}^{+} \cup\{0\}$. Moreover, for every $r \geq 0$ the functions

$$
\mathbb{S}^{d-1} \ni u \mapsto \mathbb{P}(\{\langle R, u\rangle>r\}), \text { and } \mathbb{S}^{d-1} \ni u \mapsto \mathbb{P}(\{\langle A R, u\rangle>r\}),
$$

are continuous. 
Proof At the beginning, we assume that the law $\eta$ of $B$ is nonsingular, i.e. $\left\|\eta_{s}\right\|<1$. Let $v$ be the law of $R$ and $\mu$ be the law of $A \in G$. Let $*$ be the classical convolution on $\mathbb{R}^{d}$. Moreover, we define $\xi=\mu *_{G} v$, where $\mu *_{G} v(D)=\int_{G} \int_{\mathbb{R}^{d}} \mathbf{1}_{D}(a x) v(d x) \mu(d a)$ and $D \in \mathcal{B}$ or $\left(\mathbb{R}^{d}\right)$. Obviously $\xi$ defines a probability measure on $\mathbb{R}^{d}$ which coincide with the distribution of $A R$. Notice that $\nu=\xi^{* N} * \eta$, since $R \stackrel{\mathcal{D}}{=} \sum_{i=1}^{N} A_{i} R_{i}+B$, and observe that by the Lebesgue decomposition we obtain

$$
\begin{aligned}
v_{a}+ & v_{s}=v=\left(\xi_{a}+\xi_{s}\right)^{* N} *\left(\eta_{a}+\eta_{s}\right) \\
= & \sum_{n=0}^{N}\left(\begin{array}{l}
N \\
n
\end{array}\right) \xi_{a}^{* n} * \xi_{s}^{*(N-n)} * \eta_{a}+\sum_{n=1}^{N}\left(\begin{array}{l}
N \\
n
\end{array}\right) \xi_{a}^{* n} * \xi_{s}^{*(N-n)} * \eta_{s} \\
& +\left(\xi_{s}^{* N} * \eta_{s}\right)_{a}+\left(\xi_{s}^{* N} * \eta_{s}\right)_{s},
\end{aligned}
$$

and by its uniqueness $v_{s}=\left(\xi_{s}^{* N} * \eta_{s}\right)_{s}$. This gives $\left\|v_{s}\right\| \leq\left\|\xi_{s}\right\|^{N}\left\|\eta_{s}\right\|$. Again by the Lebesgue decomposition and its uniqueness we have $\xi=\mu *_{G} v=\mu *_{G} v_{a}+\mu *_{G} v_{s}$, hence $\left\|\xi_{s}\right\|=\left\|\left(\mu *_{G} v\right)_{s}\right\| \leq\left\|\mu *_{G} v_{s}\right\| \leq\left\|v_{s}\right\|$. Now combining $\left\|v_{s}\right\| \leq\left\|\xi_{s}\right\|^{N}\left\|\eta_{s}\right\|$ and $\left\|\xi_{s}\right\| \leq\left\|v_{s}\right\|$ we get $\left\|v_{s}\right\| \leq\left\|v_{s}\right\|^{N}\left\|\eta_{s}\right\|$, if $\left\|v_{s}\right\|>0$, then $1 \leq\left\|v_{s}\right\|^{N-1}\left\|\eta_{s}\right\| \leq$ $\left\|\eta_{s}\right\|<1$. This contradiction shows that $\left\|v_{s}\right\|=0$ hence $v$ is absolutely continuous with respect to the Lebesgue measure, which in turn implies that $\mathbb{P}(\{\langle R, u\rangle=r\})=0$, for every $(u, r) \in \mathbb{S}^{+} \times \mathbb{R}^{+} \cup\{0\}$.

If the law $\eta$ of $B$ is singular, i.e. $\left\|\eta_{s}\right\|=1$, then for fixed $(u, r) \in \mathbb{S}^{+} \times \mathbb{R}^{+} \cup\{0\}$, we have $\mathbb{P}(\{\langle R, u\rangle=r\})=0$, since $\mathbb{P}(\{\langle B, u\rangle=r\})=0$.

Now we prove that $\mathbb{S}^{d-1} \ni u \mapsto \mathbb{P}(\{\langle R, u\rangle>r\})$ is continuous. Take any $\left(u_{n}\right)_{n \in \mathbb{N}} \subseteq \mathbb{S}^{+}$such that $\lim _{n \rightarrow \infty} u_{n}=u \in \mathbb{S}^{+}$and consider

$$
\begin{aligned}
\left|\mathbb{P}\left(\left\{\left\langle R, u_{n}\right\rangle>r\right\}\right)-\mathbb{P}(\{\langle R, u\rangle>r\})\right| \leq & \mathbb{P}\left(\left\{\left\langle R, u_{n}\right\rangle>r \text {, and }\langle R, u\rangle \leq r\right\}\right) \\
& +\mathbb{P}\left(\left\{\left\langle R, u_{n}\right\rangle \leq r \text {, and }\langle R, u\rangle>r\right\}\right),
\end{aligned}
$$

then

$$
\begin{aligned}
\mathbb{P}\left(\left\{\langle R, u\rangle \leq r<\left\langle R, u_{n}\right\rangle\right\}\right) & =\mathbb{P}\left(\left\{0 \leq r-\langle R, u\rangle<\left\langle R, u_{n}\right\rangle-\langle R, u\rangle\right\}\right) \\
& \leq \mathbb{P}\left(\left\{0 \leq r-\langle R, u\rangle \leq|R|\left|u_{n}-u\right|\right\}\right), \text { and } \\
\mathbb{P}\left(\left\{\left\langle R, u_{n}\right\rangle \leq r<\langle R, u\rangle\right\}\right) & =\mathbb{P}\left(\left\{\left\langle R, u_{n}\right\rangle-\langle R, u\rangle \leq r-\langle R, u\rangle<0\right\}\right) \\
& \leq \mathbb{P}\left(\left\{-|R|\left|u_{n}-u\right| \leq r-\langle R, u\rangle<0\right\}\right) .
\end{aligned}
$$

If $\left|u_{n}-u\right|<1 / m$, then

$$
\begin{aligned}
\left|\mathbb{P}\left(\left\{\left\langle R, u_{n}\right\rangle>r\right\}\right)-\mathbb{P}(\{\langle R, u\rangle>r\})\right| & \leq \mathbb{P}\left(\left\{|\langle R, u\rangle-r| \leq|R|\left|u_{n}-u\right|\right\}\right) \\
& \leq \mathbb{P}(\{|\langle R, u\rangle-r| \leq|R| / m\}) .
\end{aligned}
$$

We also know that $\lim _{m \rightarrow \infty} \mathbb{P}(\{|\langle R, u\rangle-r| \leq|R| / m\})=\mathbb{P}(\{\langle R, u\rangle=r\})=0$, hence

$$
\lim _{n \rightarrow \infty}\left|\mathbb{P}\left(\left\{\left\langle R, u_{n}\right\rangle>r\right\}\right)-\mathbb{P}(\{\langle R, u\rangle>r\})\right|=0 .
$$


The same arguments work for $u \mapsto \mathbb{P}(\{\langle A R, u\rangle>r\})$, since $A \in G$ is independent of $R$.

Lemma 5.8 Under the assumptions of Theorem 1.9, there exists $0<\beta_{1}<1$ such that for every $\beta \in\left[0, \beta_{1}\right)$, there is a finite constant $C_{\beta}>0$, such that for every $(u, t) \in \mathbb{S}^{+} \times \mathbb{R}$ we have

$$
\begin{aligned}
g_{1}(u, t) & =\frac{1}{e^{t} e_{*}^{\chi}(u)} \int_{0}^{e^{t}} r^{\chi}\left|\mathbb{P}\left(\left\{\max _{1 \leq i \leq N}\left\langle A_{i} R_{i}, u\right\rangle>r\right\}\right)-N \mathbb{P}(\{\langle A R, u\rangle>r\})\right| d r \\
& \leq C_{\beta} e^{-\beta|t|}
\end{aligned}
$$

and

$$
\begin{aligned}
& \int_{0}^{\infty}\left(N \mathbb{P}(\{\langle A R, u\rangle>r\})-\mathbb{P}\left(\left\{\max _{1 \leq i \leq N}\left\langle A_{i} R_{i}, u\right\rangle>r\right\}\right)\right) r^{\chi+\beta-1} d r \\
& =\frac{1}{\chi+\beta} \mathbb{E}\left(\sum_{i=1}^{N}\left\langle A_{i} R_{i}, u\right\rangle^{\chi+\beta}-\left(\max _{1 \leq i \leq N}\left\langle A_{i} R_{i}, u\right\rangle\right)^{\chi+\beta}\right) .
\end{aligned}
$$

Moreover, $\mathbb{S}^{+} \times \mathbb{R} \ni(u, t) \mapsto g_{1}(u, t)$ is continuous.

In the proof we extend the approach developed in [13].

Proof Let $\beta_{1} \in(0, \min \{1, \chi / 2\})$ and take any $0 \leq \beta<\beta_{1}$. Then for every $t>0$

$$
\begin{aligned}
I_{1}(t) & =e^{-\beta t} e^{-(1-\beta) t} \int_{0}^{e^{t}} r^{\chi}\left|\mathbb{P}\left(\left\{\max _{1 \leq i \leq N}\left\langle A_{i} R_{i}, u\right\rangle>r\right\}\right)-N \mathbb{P}(\{\langle A R, u\rangle>r\})\right| d r \\
& \leq e^{-\beta t} \int_{0}^{e^{t}} r^{\chi+\beta-1}\left|\mathbb{P}\left(\left\{\max _{1 \leq i \leq N}\left\langle A_{i} R_{i}, u\right\rangle>r\right\}\right)-N \mathbb{P}(\{\langle A R, u\rangle>r\})\right| d r .
\end{aligned}
$$

Now observe that $N \mathbb{P}(\{\langle A R, u\rangle>r\}) \geq \mathbb{P}\left(\left\{\max _{1 \leq i \leq N}\left\langle A_{i} R_{i}, u\right\rangle>r\right\}\right)$, then

$$
\begin{aligned}
& \int_{0}^{1}\left(N \mathbb{P}(\{\langle A R, u\rangle>r\})-\mathbb{P}\left(\left\{\max _{1 \leq i \leq N}\left\langle A_{i} R_{i}, u\right\rangle>r\right\}\right)\right) r^{\chi+\beta-1} d r \\
& \quad \leq N \int_{0}^{1} r^{\chi+\beta-1} d r<\infty .
\end{aligned}
$$

Let us define $\bar{F}(y)=\mathbb{P}(\{\langle A R, u\rangle>y\})$, and $\gamma=\chi+\beta-\beta_{1}$, and notice $N \mathbb{P}(\{\langle A R, u\rangle>r\})-\mathbb{P}\left(\left\{\max _{1 \leq i \leq N}\left\langle A_{i} R_{i}, u\right\rangle>r\right\}\right)=(1-\bar{F}(r))^{N}-1+N \bar{F}(r) \leq$ $e^{-N \bar{F}(r)}-1+N \bar{F}(r)$, and for some $c>0$ 


$$
\bar{F}(r)=\mathbb{P}(\{\langle A R, u\rangle>r\}) \leq r^{-\gamma} \mathbb{E}\left(\langle A R, u\rangle^{\gamma}\right) \leq c r^{-\gamma}
$$

Clearly, $1<\frac{\chi+\beta}{\gamma}$, and $\beta_{1}<\chi / 2$ implies $\gamma=\chi+\beta-\beta_{1} \geq \chi / 2+\beta / 2$, hence $\frac{\chi+\beta}{\gamma}<2$. Then

$$
\begin{aligned}
\int_{1}^{\infty} & \left(N \mathbb{P}(\{\langle A R, u\rangle>r\})-\mathbb{P}\left(\left\{\max _{1 \leq i \leq N}\left\langle A_{i} R_{i}, u\right\rangle>r\right\}\right)\right) r^{\chi+\beta-1} d r \\
& \leq \int_{1}^{\infty}\left(e^{-N \bar{F}(r)}-1+N \bar{F}(r)\right) r^{\chi+\beta-1} d r \leq \int_{1}^{\infty}\left(e^{-c N r^{-\gamma}}-1+c N r^{-\gamma}\right) r^{\chi+\beta-1} d r \\
& =\int_{1}^{\infty}\left(e^{-c N r^{-\gamma}}-1+c N r^{-\gamma}\right)\left(\left(c N r^{-\gamma}\right) \frac{1}{c N}\right)^{-\frac{\chi+\beta}{\gamma}} \frac{d r}{r} \\
= & \frac{(c N)^{\frac{\chi+\beta}{\gamma}}}{\gamma} \int_{0}^{c N}\left(e^{-r}-1+r\right) r^{-\frac{\chi+\beta}{\gamma}-1} d r \leq \frac{(c N)^{\frac{\chi x+\beta}{\gamma}}}{\gamma} \int_{0}^{\infty}\left(e^{-r}-1+r\right) r^{-\frac{\chi+\beta}{\gamma}-1} d r \\
\leq & \frac{(c N)^{\frac{\chi+\beta}{\gamma}}}{\gamma}\left(\frac{1}{2} \int_{0}^{1-\frac{\chi+\beta}{\gamma}} d r+\int_{1}^{\infty} r^{-\frac{\chi+\beta}{\gamma}} d r\right) \\
= & \frac{(c N)^{\frac{\chi+\beta}{\gamma}}}{\gamma}\left(\frac{1}{2\left(2-\frac{\chi+\beta}{\gamma}\right)}+\frac{1}{\frac{\chi+\beta}{\gamma}-1}\right)<\infty .
\end{aligned}
$$

We have shown that $I_{1}(t) \leq C_{\beta} e^{-\beta t}$, for every $\beta \in\left[0, \beta_{1}\right)$ and $t \geq 0$ with the constant $C_{\beta}>0$ which does not depend on $u \in \mathbb{S}^{+}$. If is not difficult to see that the statement is clear for $t \leq 0$. A straightforward applications of Fubini theorem yields

$$
\begin{aligned}
& \int_{0}^{\infty}\left(N \mathbb{P}(\{\langle A R, u\rangle>r\})-\mathbb{P}\left(\left\{\max _{1 \leq i \leq N}\left\langle A_{i} R_{i}, u\right\rangle>r\right\}\right)\right) r^{\chi+\beta-1} d r \\
& \quad=\int_{0}^{\infty}\left(\mathbb{E}\left(\sum_{i=1}^{N} \mathbf{1}_{\left\{\left\langle A_{i} R_{i}, u\right\rangle>r\right\}}\right)-\mathbb{E}\left(\mathbf{1}_{\left\{\max _{1 \leq i \leq N}\left\langle A_{i} R_{i}, u\right\rangle>r\right\}}\right)\right) r^{\chi+\beta-1} d r \\
& \quad=\mathbb{E}\left(\int_{0}^{\infty}\left(\sum_{i=1}^{N} \mathbf{1}_{\left\{\left\langle A_{i} R_{i}, u\right\rangle>r\right\}}-\mathbf{1}_{\left\{\max _{1 \leq i \leq N}\left\langle A_{i} R_{i}, u\right\rangle>r\right\}}\right) r^{\chi+\beta-1} d r\right)
\end{aligned}
$$




$$
\begin{aligned}
& =\mathbb{E}\left(\sum_{i=1}^{N} \int_{0}^{\left\langle A_{i} R_{i}, u\right\rangle} r^{\chi+\beta-1} d r-\int_{0}^{\max _{1 \leq i \leq N}\left\langle A_{i} R_{i}, u\right\rangle} r^{\chi+\beta-1} d r\right) \\
& =\frac{1}{\chi+\beta} \mathbb{E}\left(\sum_{i=1}^{N}\left\langle A_{i} R_{i}, u\right\rangle^{\chi+\beta}-\left(\max _{1 \leq i \leq N}\left\langle A_{i} R_{i}, u\right\rangle\right)^{\chi+\beta}\right) .
\end{aligned}
$$

In order to show the continuity of $\mathbb{S}^{+} \times \mathbb{R} \ni(u, t) \mapsto g_{1}(u, t)$ it is enough to prove the continuity of

$$
u \mapsto \frac{1}{e^{t}} \int_{0}^{e^{t}} r^{\chi}\left(N \mathbb{P}(\{\langle A R, u\rangle>r\})-\mathbb{P}\left(\left\{\max _{1 \leq i \leq N}\left\langle A_{i} R_{i}, u\right\rangle>r\right\}\right)\right) d r
$$

In this purpose observe that $\mathbb{P}\left(\left\{\max _{1 \leq i \leq N}\left\langle A_{i} R_{i}, u\right\rangle>r\right\}\right)=1-(1-\mathbb{P}(\{\langle A R, u\rangle>$ $r\}))^{N}=1-(1-\bar{F}(r))^{N}$, where $\bar{F}(y)=\mathbb{P}(\{\langle A R, u\rangle>y\})$, hence Lemma 5.7 guarantees that

$$
\begin{aligned}
u & \mapsto N \mathbb{P}(\{\langle A R, u\rangle>r\})-\mathbb{P}\left(\left\{\max _{1 \leq i \leq N}\left\langle A_{i} R_{i}, u\right\rangle>r\right\}\right) \\
& =(1-\bar{F}(r))^{N}-1+N \bar{F}(r),
\end{aligned}
$$

is continuous. Observe that

$$
\begin{aligned}
N \mathbb{P}(\{\langle A R, u\rangle>r\})-\mathbb{P}\left(\left\{\max _{1 \leq i \leq N}\left\langle A_{i} R_{i}, u\right\rangle>r\right\}\right) \\
\leq \begin{cases}N, & \text { if } r \leq 1, \\
e^{-N \bar{F}(r)}-1+N \bar{F}(r), & \text { if } r>1,\end{cases}
\end{aligned}
$$

then arguing in a similar way as above with $\beta=0$, and using Lebesgue dominated convergence theorem we obtain the continuity of (5.11) and the lemma follows.

Now we are going to prove inequality (5.13) and (5.15), that will provide necessary estimates for Lemma 5.16. The first one was proved in [13] and was sufficient in the one dimensional case discussed there. The second one is more subtle and allows us to deal with our situation.

Here and subsequently $\lceil\alpha\rceil$ denotes the smallest integer $\geq \alpha$.

Lemma 5.12 Let $\alpha>1$ and $p=\lceil\alpha\rceil \geq 2$. For any sequence of nonnegative i.i.d. random variables $Y, Y_{1}, Y_{2}, \ldots$ such that $\mathbb{E}\left(Y^{p-1}\right)<\infty$, and any $k \in \mathbb{N}$ we have

$$
\mathbb{E}\left(\left(\sum_{i=1}^{k} Y_{i}\right)^{\alpha}-\sum_{i=i}^{k} Y_{i}^{\alpha}\right) \leq k^{\alpha} \mathbb{E}\left(Y^{p-1}\right)^{\frac{\alpha}{p-1}}
$$


Proof As mentioned before the proof is contained in [13].

Lemma 5.14 Let $p \in \mathbb{N}$ and $\beta \in(0,1)$. Then for any $\delta \in\left(0, \frac{p(1-\beta)}{p+1}\right)$, for any sequence of nonnegative i.i.d. random variables $Y, Y_{1}, Y_{2}, \ldots$ such that $\mathbb{E}\left(Y^{p-\delta}\right)<$ $\infty$, and any $k \in \mathbb{N}$ we have

$$
\mathbb{E}\left(\left(\sum_{i=1}^{k} Y_{i}\right)^{p+\beta}-\sum_{i=1}^{k} Y_{i}^{p+\beta}\right) \leq k^{p+1} \mathbb{E}\left(Y^{p-\delta}\right)^{\frac{p+\beta}{p-\delta}}
$$

Proof Define $A_{p}(k)=\left\{\left(j_{1}, \ldots, j_{k}\right) \in \mathbb{Z}^{k}: j_{1}+\cdots+j_{k}=p\right.$, and $\left.0 \leq j_{i}<p\right\}$ and observe that

$$
\begin{aligned}
\left(\sum_{i=1}^{k} Y_{i}\right)^{p-\delta} & =\left(\left(\sum_{i=1}^{k} Y_{i}\right)^{p}\right)^{\frac{p-\delta}{p}} \\
& =\left(\sum_{i=1}^{k} Y_{i}^{p}+\sum_{\left(j_{1}, \ldots, j_{k}\right) \in A_{p}(k)}\left(\begin{array}{c}
p \\
j_{1}, \ldots, j_{k}
\end{array}\right) Y_{1}^{j_{1}} \ldots Y_{k}^{j_{k}}\right)^{\frac{p-\delta}{p}} \\
& \leq \sum_{i=1}^{k} Y_{i}^{p-\delta}+\sum_{\left(j_{1}, \ldots, j_{k}\right) \in A_{p}(k)}\left(\begin{array}{c}
p \\
j_{1}, \ldots, j_{k}
\end{array}\right)\left(Y_{1}^{j_{1}} \ldots Y_{k}^{j_{k}}\right)^{\frac{p-\delta}{p}}
\end{aligned}
$$

Now observe that $\beta+\delta<\beta+\frac{p(1-\beta)}{p+1}<1$. By the above inequality

$$
\begin{aligned}
& \left(\sum_{i=1}^{k} Y_{i}\right)^{p+\beta}=\left(\sum_{i=1}^{k} Y_{i}\right)^{p-\delta}\left(\sum_{i=1}^{k} Y_{i}\right)^{\beta+\delta} \\
& =\left(\left(\sum_{i=1}^{k} Y_{i}\right)^{p-\delta}-\sum_{i=1}^{k} Y_{i}^{p-\delta}\right)\left(\sum_{i=1}^{k} Y_{i}\right)^{\beta+\delta} \\
& +\left(\sum_{i=1}^{k} Y_{i}^{p-\delta}\right)\left(\sum_{i=1}^{k} Y_{i}\right)^{\beta+\delta} \\
& \leq\left(\sum_{\left(j_{1}, \ldots, j_{k}\right) \in A_{p}(k)}\left(\begin{array}{c}
p \\
j_{1}, \ldots, j_{k}
\end{array}\right)\left(Y_{1}^{j_{1}} \ldots . Y_{k}^{j_{k}}\right)^{\frac{p-\delta}{p}}\right)\left(\sum_{i=1}^{k} Y_{i}^{\beta+\delta}\right) \\
& +\left(\sum_{i=1}^{k} Y_{i}^{p-\delta}\right)\left(\sum_{i=1}^{k} Y_{i}^{\beta+\delta}\right) .
\end{aligned}
$$


It follows that

$$
\begin{aligned}
\left(\sum_{i=1}^{k} Y_{i}\right)^{p+\beta}-\sum_{i=1}^{k} Y_{i}^{p+\beta} & \leq \sum_{i=1}^{k} \sum_{\left(j_{1}, \ldots, j_{k}\right) \in A_{p}(k)}\left(\begin{array}{c}
p \\
j_{1}, \ldots, j_{k}
\end{array}\right)\left(Y_{1}^{j_{1}} \cdots Y_{k}^{j_{k}}\right)^{\frac{p-\delta}{p}} Y_{i}^{\beta+\delta} \\
& +\sum_{i \neq j} Y_{i}^{p-\delta} Y_{j}^{\beta+\delta} .
\end{aligned}
$$

But $j_{i} \leq p-1$. Hence $\frac{j_{i}(p-\delta)}{p}+\beta+\delta \leq \frac{1}{p}(p-1)(p-\delta)+\beta+\delta \leq p+\beta-1+\frac{\delta}{p}<p-\delta$, since

$$
\begin{aligned}
0<\delta<\frac{p(1-\beta)}{p+1} & \Longrightarrow \delta\left(1+\frac{1}{p}\right)<1-\beta \Longrightarrow \beta-1+\frac{\delta}{p}<-\delta \\
& \Longrightarrow p+\beta-1+\frac{\delta}{p}<p-\delta .
\end{aligned}
$$

Now we have

$$
\begin{aligned}
& \mathbb{E}\left(Y_{1}^{\frac{j_{1}(p-\delta)}{p}} \cdots Y_{i}^{\frac{j_{i}(p-\delta)}{p}+\beta+\delta} \cdots Y_{k}^{\frac{j_{k}(p-\delta)}{p}}\right) \\
& \leq\|Y\|_{p-\delta}^{\frac{j_{1}(p-\delta)}{p}} \cdots \cdots\|Y\|_{p-\delta}^{\frac{j_{i}(p-\delta)}{p}+\beta+\delta} \cdots \cdots \|_{p-\delta}^{\frac{j_{k}(p-\delta)}{p}} \\
& =\|Y\|_{p-\delta}^{p+\beta}
\end{aligned}
$$

because $j_{1}+\cdots+j_{k}=p$. Observe that

$$
\delta<\frac{p(1-\beta)}{p+1} \Longrightarrow \delta<\frac{p-\beta}{2} \Longrightarrow \beta+\delta<p-\delta,
$$

hence $\mathbb{E}\left(Y_{i}^{p-\delta} Y_{j}^{\beta+\delta}\right)=\|Y\|_{p-\delta}^{p-\delta}\|Y\|_{\beta+\delta}^{\beta+\delta} \leq\|Y\|_{p-\delta}^{p+\beta}$, and so

$$
\begin{aligned}
& \mathbb{E}\left(\left(\sum_{i=1}^{k} Y_{i}\right)^{p+\beta}-\sum_{i=1}^{k} Y_{i}^{p+\beta}\right) \leq k\left(k^{p}-k\right) \mathbb{E}\left(Y^{p-\delta}\right)^{\frac{p+\beta}{p-\delta}}+k^{2} \mathbb{E}\left(Y^{p-\delta}\right)^{\frac{p+\beta}{p-\delta}} \\
& =k^{p+1} \mathbb{E}\left(Y^{p-\delta}\right)^{\frac{p+\beta}{p-\delta}}
\end{aligned}
$$

Lemma 5.16 Under the assumptions of Theorem 1.9, there exists $0<\beta_{2}<1$ such that for every $\beta \in\left[0, \beta_{2}\right)$, there is a finite constant $C_{\beta}>0$, such that for every $(u, t) \in \mathbb{S}^{+} \times \mathbb{R}$ we have 


$$
\begin{aligned}
g_{2}(u, t) & =\frac{1}{e^{t} e_{*}^{\chi}(u)} \int_{0}^{e^{t}} r^{\chi}\left|\mathbb{P}(\{\langle R, u\rangle>r\})-\mathbb{P}\left(\left\{\max _{1 \leq i \leq N}\left\langle A_{i} R_{i}, u\right\rangle>r\right\}\right)\right| d r \\
& \leq C_{\beta} e^{-\beta|t|}
\end{aligned}
$$

and

$$
\begin{aligned}
& \int_{0}^{\infty} r^{\chi+\beta-1}\left(\mathbb{P}(\{\langle R, u\rangle>r\})-\mathbb{P}\left(\left\{\max _{1 \leq i \leq N}\left\langle A_{i} R_{i}, u\right\rangle>r\right\}\right)\right) d r \\
& =\frac{1}{\chi+\beta} \mathbb{E}\left(\langle R, u\rangle^{\chi+\beta}-\left(\max _{1 \leq i \leq N}\left\langle A_{i} R_{i}, u\right\rangle\right)^{\chi+\beta}\right) .
\end{aligned}
$$

Moreover, $\mathbb{S}^{+} \times \mathbb{R} \ni(u, t) \mapsto g_{2}(u, t)$ is continuous.

Proof Let $0<\beta_{2}<\min \left\{\varepsilon, \beta_{1}\right\}\left(\varepsilon>0\right.$ as in Theorem 1.9 and $\beta_{1}>0$ as in Lemma 5.8) and take $\beta \in\left[0, \beta_{2}\right)$. Then for every $t>0$

$$
\begin{aligned}
I_{2}(t) & =e^{-\beta t} e^{-(1-\beta) t} \int_{0}^{e^{t}} r^{\chi}\left|\mathbb{P}(\{\langle R, u\rangle>r\})-\mathbb{P}\left(\left\{\max _{1 \leq i \leq N}\left\langle A_{i} R_{i}, u\right\rangle>r\right\}\right)\right| d r \\
& \leq e^{-\beta t} \int_{0}^{\infty} r^{\chi+\beta-1}\left|\mathbb{P}(\{\langle R, u\rangle>r\})-\mathbb{P}\left(\left\{\max _{1 \leq i \leq N}\left\langle A_{i} R_{i}, u\right\rangle>r\right\}\right)\right| d r .
\end{aligned}
$$

Observe that $\langle R, u\rangle \geq \max _{1 \leq i \leq N}\left\langle A_{i} R_{i}, u\right\rangle$. Then applying Fubini theorem as in Lemma 5.8 we obtain

$$
\begin{gathered}
\int_{0}^{\infty} r^{\chi+\beta-1}\left(\mathbb{P}(\{\langle R, u\rangle>r\})-\mathbb{P}\left(\left\{\max _{1 \leq i \leq N}\left\langle A_{i} R_{i}, u\right\rangle>r\right\}\right)\right) d r \\
=\frac{1}{\chi+\beta} \mathbb{E}\left(\langle R, u\rangle^{\chi+\beta}-\left(\max _{1 \leq i \leq N}\left\langle A_{i} R_{i}, u\right\rangle\right)^{\chi+\beta}\right) .
\end{gathered}
$$

If $0<\chi<1$, take any $\beta \in\left[0, \beta_{2}\right)$ such that $0<\chi+\beta \leq 1$ and notice

$$
\begin{aligned}
& \mathbb{E}\left(\langle R, u\rangle^{\chi+\beta}-\left(\max _{1 \leq i \leq N}\left\langle A_{i} R_{i}, u\right\rangle\right)^{\chi+\beta}\right) \\
& \leq \mathbb{E}\left(\langle B, u\rangle^{\chi+\beta}\right)+\mathbb{E}\left(\sum_{i=1}^{N}\left\langle A_{i} R_{i}, u\right\rangle^{\chi+\beta}-\left(\max _{1 \leq i \leq N}\left\langle A_{i} R_{i}, u\right\rangle\right)^{\chi+\beta}\right)<\infty
\end{aligned}
$$

since $\mathbb{E}\left(|B|^{\chi+\varepsilon}\right)<\infty$ for some $\varepsilon>0$, and the second term is finite by Lemma 5.8. 
If $\chi \geq 1$ we write

$$
\begin{aligned}
& \mathbb{E}\left(\langle R, u\rangle^{\chi+\beta}-\left(\max _{1 \leq i \leq N}\left\langle A_{i} R_{i}, u\right\rangle\right)^{\chi+\beta}\right) \\
& =\mathbb{E}\left(\langle R, u\rangle^{\chi+\beta}-\sum_{i=1}^{N}\left\langle A_{i} R_{i}, u\right\rangle^{\chi+\beta}\right) \\
& \quad+\mathbb{E}\left(\sum_{i=1}^{N}\left\langle A_{i} R_{i}, u\right\rangle^{\chi+\beta}-\left(\max _{1 \leq i \leq N}\left\langle A_{i} R_{i}, u\right\rangle\right)^{\chi+\beta}\right) .
\end{aligned}
$$

We have to estimate only the first term, since the second one is finite by Lemma 5.8. In this purpose we use Lemmas 5.12 and 5.14. Notice that

$$
\begin{aligned}
& \mathbb{E}\left(\langle R, u\rangle^{\chi+\beta}-\sum_{i=1}^{N}\left\langle A_{i} R_{i}, u\right\rangle^{\chi+\beta}\right) \\
& =\mathbb{E}\left(\left\langle\sum_{i=1}^{N} A_{i} R_{i}+B, u\right\rangle^{\chi+\beta}-\left\langle\sum_{i=1}^{N} A_{i} R_{i}, u\right\rangle^{\chi+\beta}\right) \\
& +\mathbb{E}\left(\left\langle\sum_{i=1}^{N} A_{i} R_{i}, u\right\rangle^{\chi+\beta}-\sum_{i=1}^{N}\left\langle A_{i} R_{i}, u\right\rangle^{\chi+\beta}\right) \\
& \leq(\chi+\beta) \mathbb{E}\left(|B|\left(\sum_{i=1}^{N}\left|A_{i} R_{i}\right|+|B|\right)^{\chi+\beta-1}\right) \\
& +\mathbb{E}\left(\left\langle\sum_{i=1}^{N} A_{i} R_{i}, u\right\rangle^{\chi+\beta}-\sum_{i=1}^{N}\left\langle A_{i} R_{i}, u\right\rangle^{\chi+\beta}\right) .
\end{aligned}
$$

$\mathbb{E}\left(|B|\left(\sum_{i=1}^{N}\left|A_{i} R_{i}\right|+|B|\right)^{\chi+\beta-1}\right)$ is finite, since $\mathbb{E}\left(\|A\|^{\chi+\beta-1}\right)<\infty, \mathbb{E}\left(|B|^{\chi+\varepsilon}\right)$ $<\infty$ and Theorem 1.7 yields $\mathbb{E}\left(|R|^{\chi+\beta-1}\right)<\infty$.

If $\chi \notin \mathbb{N}$ we assume additionally that $\left\lceil\chi+\beta_{2}\right\rceil=\lceil\chi\rceil$, (which holds for sufficiently small $\beta_{2}>0$ ). Applying inequality (5.13) with $p=\lceil\chi\rceil=\lceil\chi+\beta\rceil$ and $\beta \in\left[0, \beta_{2}\right)$ we obtain

$\mathbb{E}\left(\left\langle\sum_{i=1}^{N} A_{i} R_{i}, u\right\rangle^{\chi+\beta}-\sum_{i=1}^{N}\left\langle A_{i} R_{i}, u\right\rangle^{\chi+\beta}\right) \leq N^{\chi+\beta}\left(\mathbb{E}\left(\langle A R, u\rangle^{p-1}\right)\right)^{\frac{\chi+\beta}{p-1}}<\infty$

since $p-1<\chi$. 
If $\chi \in \mathbb{N}$ and $\beta \in\left[0, \beta_{2}\right)$ take any $\delta \in\left(0, \frac{p(1-\beta)}{p+1}\right)$ as in Lemma 5.14 with $p=\chi$, then by inequality (5.15) we get

$\mathbb{E}\left(\left\langle\sum_{i=1}^{N} A_{i} R_{i}, u\right\rangle^{\chi+\beta}-\sum_{i=1}^{N}\left\langle A_{i} R_{i}, u\right\rangle^{\chi+\beta}\right) \leq N^{\chi+1}\left(\mathbb{E}\left(\langle A R, u\rangle^{\chi-\delta}\right)\right)^{\frac{\chi+\beta}{\chi-\delta}}<\infty$

Finally, we have proved $I_{2}(t) \leq C_{\beta} e^{-\beta|t|}$, for every $\beta \in\left[0, \beta_{2}\right)$ and $t>0$ with $C_{\beta}<\infty$ independent of $u \in \mathbb{S}^{+}$. If $t \leq 0$ there is nothing to do and the statement follows.

It remains to prove that $\mathbb{S}^{+} \times \mathbb{R} \ni(u, t) \mapsto g_{2}(u, t)$ is continuous. In this purpose it suffices to show continuity of

$$
u \mapsto \frac{1}{e^{t}} \int_{0}^{e^{t}} r^{\chi}\left(\mathbb{P}(\{\langle R, u\rangle>r\})-\mathbb{P}\left(\left\{\max _{1 \leq i \leq N}\left\langle A_{i} R_{i}, u\right\rangle>r\right\}\right)\right) d r
$$

Observe that

$$
\begin{aligned}
\frac{1}{e^{t}} \int_{0}^{e^{t}} r^{\chi} \mid \mathbb{P}\left(\left\{\left\langle R, u_{n}\right\rangle>r\right\}\right)-\mathbb{P}\left(\left\{\max _{1 \leq i \leq N}\left\langle A_{i} R_{i}, u_{n}\right\rangle>r\right\}\right) \\
\quad-\left(\mathbb{P}\left(\left\{\left\langle R, u_{0}\right\rangle>r\right\}\right)-\mathbb{P}\left(\left\{\max _{1 \leq i \leq N}\left\langle A_{i} R_{i}, u_{0}\right\rangle>r\right\}\right)\right) \mid d r \\
\leq \int_{0}^{\infty} r^{\chi-1} \mid \mathbb{P}\left(\left\{\left\langle R, u_{n}\right\rangle>r\right\}\right)-\mathbb{P}\left(\left\{\max _{1 \leq i \leq N}\left\langle A_{i} R_{i}, u_{n}\right\rangle>r\right\}\right) \\
\quad-\left(\mathbb{P}\left(\left\{\left\langle R, u_{0}\right\rangle>r\right\}\right)-\mathbb{P}\left(\left\{\max _{1 \leq i \leq N}\left\langle A_{i} R_{i}, u_{0}\right\rangle>r\right\}\right)\right) \mid d r .
\end{aligned}
$$

It is enough to show that the last integral converges to 0 as $\lim _{n \rightarrow \infty} u_{n}=u_{0}$. In this purpose we will use an extended version of Lebesgue dominated convergence theorem (see for instance in [1]). Namely,

Theorem 5.20 Given a measure space $(X, \mathcal{M}, \mu)$ (where $\mu$ may take values in $[0, \infty])$. Let $\left(f_{n}\right)_{n \in \mathbb{N}}$ and $\left(h_{n}\right)_{n \in \mathbb{N}}, f$ and $h$ be $\mathcal{M}$ measurable, real valued functions on X. Suppose

- $\lim _{n \rightarrow \infty} f_{n}=f$ and $\lim _{n \rightarrow \infty} h_{n}=h$ a.e. on $X$,

- $\left(h_{n}\right)_{n \in \mathbb{N}}$ and $h$ are all $\mu$ integrable on $X$ and $\lim _{n \rightarrow \infty} \int_{X} h_{n} d \mu=\int_{X} h d \mu$,

- $\left|f_{n}\right| \leq h_{n}$ a.e. on $X$ for every $n \in \mathbb{N}$.

Then $f$ is $\mu$ integrable on $X$ and $\lim _{n \rightarrow \infty} \int_{X} f_{n} d \mu=\int_{X} f d \mu$. 
We will apply Theorem 5.20 with

$$
\begin{aligned}
f_{n}(r)= & r^{\chi-1} \mid \mathbb{P}\left(\left\{\left\langle R, u_{n}\right\rangle>r\right\}\right)-\mathbb{P}\left(\left\{\max _{1 \leq i \leq N}\left\langle A_{i} R_{i}, u_{n}\right\rangle>r\right\}\right) \\
& -\left(\mathbb{P}\left(\left\{\left\langle R, u_{0}\right\rangle>r\right\}\right)-\mathbb{P}\left(\left\{\max _{1 \leq i \leq N}\left\langle A_{i} R_{i}, u_{0}\right\rangle>r\right\}\right)\right), \\
h_{n}(r)= & r^{\chi-1}\left(\mathbb{P}\left(\left\{\left\langle R, u_{n}\right\rangle>r\right\}\right)-\mathbb{P}\left(\left\{\max _{1 \leq i \leq N}\left\langle A_{i} R_{i}, u_{n}\right\rangle>r\right\}\right)\right. \\
& \left.+\left(\mathbb{P}\left(\left\{\left\langle R, u_{0}\right\rangle>r\right\}\right)-\mathbb{P}\left(\left\{\max _{1 \leq i \leq N}\left\langle A_{i} R_{i}, u_{0}\right\rangle>r\right\}\right)\right)\right),
\end{aligned}
$$

and

$$
h(r)=2 r^{\chi-1}\left(\mathbb{P}\left(\left\{\left\langle R, u_{0}\right\rangle>r\right\}\right)-\mathbb{P}\left(\left\{\max _{1 \leq i \leq N}\left\langle A_{i} R_{i}, u_{0}\right\rangle>r\right\}\right)\right) .
$$

Clearly, $\left|f_{n}\right| \leq h_{n}$ for every $n \in \mathbb{N}$, and $\left(h_{n}\right)_{n \in \mathbb{N}}$ and $h$ are all integrable. Lemma 5.7 guarantees that $\lim _{n \rightarrow \infty} f_{n}(r)=0$ and $\lim _{n \rightarrow \infty} h_{n}(r)=h(r)$. In order to show that $\lim _{n \rightarrow \infty} \int_{0}^{\infty} h_{n}(r) d r=\int_{0}^{\infty} h(r) d r$, notice that by (5.18) with $\beta=0$ we have to show that

$$
\begin{gathered}
\lim _{n \rightarrow \infty} \mathbb{E}\left(\left\langle R, u_{n}\right\rangle^{\chi}-\left(\max _{1 \leq i \leq N}\left\langle A_{i} R_{i}, u_{n}\right\rangle\right)^{\chi}\right) \\
=\mathbb{E}\left(\left\langle R, u_{0}\right\rangle^{\chi}-\left(\max _{1 \leq i \leq N}\left\langle A_{i} R_{i}, u_{0}\right\rangle\right)^{\chi}\right) .
\end{gathered}
$$

But in view of the first part of this lemma and the estimates given there (5.21) is a simple consequence of a classical Lebesgue dominated convergence theorem. This finishes the proof of Lemma 5.16.

Proof of Theorem 1.9 From Lemma 5.2 we know that

$$
G(u, t)=\sum_{n=0}^{\infty} \Theta^{n} g(u, t)
$$

where

$$
g(u, t)=\frac{1}{e^{t} e_{*}^{\chi}(u)} \int_{0}^{e^{t}} r^{\chi}(\mathbb{P}(\{\langle R, u\rangle>r\})-N \mathbb{P}(\{\langle A R, u\rangle>r\})) d r .
$$

As a consequence of Lemmas 5.8 and 5.16 the function $\mathbb{S}^{+} \times \mathbb{R} \ni(u, t) \mapsto g(u, t)$ is jointly continuous. Moreover, it is possible to find $\beta>0$ and a positive constant $C_{\beta}<\infty$ such that 


$$
|g(u, t)| \leq C_{\beta} e^{-\beta|t|}, \quad \text { for every }(u, t) \in \mathbb{S}^{+} \times \mathbb{R},
$$

since $|g(u, t)| \leq g_{1}(u, t)+g_{2}(u, t)$, for $g_{1}(u, t)$ and $g_{2}(u, t)$ defined in Lemmas 5.8 and 5.16, respectively. This shows that $g(u, t)$ satisfies condition (4.24). By the Kesten's renewal theorem 4.19 we obtain

$$
\begin{aligned}
\lim _{t \rightarrow \infty} G(u, t) & =\lim _{t \rightarrow \infty} \mathbb{E}_{x}^{\chi, *}\left(\sum_{n=0}^{\infty} g\left(X_{n}, t-V_{n}\right)\right) \\
& =\frac{1}{\alpha(\chi)} \int_{\mathbb{S}^{+}}\left(\int_{\mathbb{R}} g(y, x) d x\right) \pi_{*}^{\chi}(d y)=C_{\chi} .
\end{aligned}
$$

In other words we have proved that for every $u \in \mathbb{S}^{+}$

$$
\lim _{t \rightarrow \infty} G(u, t)=\lim _{t \rightarrow \infty} \frac{1}{e^{t} e_{*}^{\chi}(u)} \int_{0}^{e^{t}} r^{\chi} \mathbb{P}(\{\langle R, u\rangle>r\}) d r=C_{\chi} \geq 0 .
$$

Hence in view of Lemma 9.3 of [5], for every $u \in \mathbb{S}^{+}$

$$
\lim _{t \rightarrow \infty} t^{\chi} \mathbb{P}(\{\langle R, u\rangle>t\})=C_{\chi} e_{*}^{\chi}(u) .
$$

It remains to prove that $C_{\chi}>0$ for every $\chi \geq 1$. (It is worth emphasizing, as we mentioned in the discussion given after Theorem 1.9, that the case when $\chi<1$ is unavailable at the moment. However, some positive results in this direction can be found in [4].) In this purpose notice that

$$
\begin{aligned}
C_{\chi} & =\frac{1}{\alpha(\chi)} \int_{\mathbb{S}^{+}}\left(\int_{\mathbb{R}} g(u, t) d t\right) \pi_{*}^{\chi}(d u) \\
& =\frac{1}{\alpha(\chi)} \int_{\mathbb{S}^{+}} \int_{\mathbb{R}}\left(\frac{1}{e^{t} e_{*}^{\chi}(u)} \int_{0}^{e^{t}} r^{\chi}(\mathbb{P}(\{\langle R, u\rangle>r\})-N \mathbb{P}(\{\langle A R, u\rangle>r\})) d r\right) d t \pi_{*}^{\chi}(d u) \\
& =\frac{1}{\alpha(\chi)} \int_{\mathbb{S}^{+}} \int_{\mathbb{R}}\left(\frac{1}{e^{t} e_{*}^{\chi}(u)} \int_{-\infty}^{t} e^{s(\chi+1)}\left(\mathbb{P}\left(\left\{\langle R, u\rangle>e^{s}\right\}\right)-N \mathbb{P}\left(\left\{\langle A R, u\rangle>e^{s}\right\}\right)\right) d s\right) d t \pi_{*}^{\chi}(d u) \\
& =\frac{1}{\alpha(\chi)} \int_{\mathbb{S}^{+}} \int_{\mathbb{R}} \int_{s}^{\infty}\left(\frac{e^{s(\chi+1)}}{e^{t} e_{*}^{\chi}(u)}\left(\mathbb{P}\left(\left\{\langle R, u\rangle>e^{s}\right\}\right)-N \mathbb{P}\left(\left\{\langle A R, u\rangle>e^{s}\right\}\right)\right) d t\right) d s \pi_{*}^{\chi}(d u) \\
& =\frac{1}{\alpha(\chi)} \int_{\mathbb{S}^{+}} \int_{\mathbb{R}} \frac{e^{s \chi}}{e_{*}^{\chi}(u)}\left(\mathbb{P}\left(\left\{\langle R, u\rangle>e^{s}\right\}\right)-N \mathbb{P}\left(\left\{\langle A R, u\rangle>e^{s}\right\}\right)\right) d s \pi_{*}^{\chi}(d u) \\
& =\frac{1}{\alpha(\chi)} \int_{\mathbb{S}^{+}} \frac{1}{e_{*}^{\chi}(u)} \int_{0}^{\infty} r^{\chi-1}(\mathbb{P}(\{\langle R, u\rangle>r\})-N \mathbb{P}(\{\langle A R, u\rangle>r\})) d r \pi_{*}^{\chi}(d u)
\end{aligned}
$$




$$
\begin{aligned}
& =\frac{1}{\alpha(\chi)} \int_{\mathbb{S}^{+}} \frac{1}{e_{*}^{\chi}(u)} \int_{0}^{\infty} \mathbb{E}\left(\mathbf{1}_{\left\{\left\langle\sum_{i=1}^{N} A_{i} R_{i}+B, u\right\rangle>r\right\}}-\sum_{i=1}^{N} \mathbf{1}_{\left\{\left\langle A_{i} R_{i}, u\right\rangle>r\right\}}\right) r^{\chi-1} d r \pi_{*}^{\chi}(d u) \\
& =\frac{1}{\alpha(\chi) \chi} \int_{\mathbb{S}^{+}} \frac{1}{e_{*}^{\chi}(u)} \mathbb{E}\left(\left\langle\sum_{i=1}^{N} A_{i} R_{i}+B, u\right\rangle^{\chi}-\sum_{i=1}^{N}\left\langle A_{i} R_{i}, u\right\rangle^{\chi}\right) \pi_{*}^{\chi}(d u) \\
& \geq \frac{1}{\alpha(\chi) \chi} \int_{\mathbb{S}^{+}} \frac{1}{e_{*}^{\chi}(u)} \mathbb{E}\left(\langle B, u\rangle^{\chi}\right) \pi_{*}^{\chi}(d u),
\end{aligned}
$$

since we have used (the fact that we are working with positive matrices and $\chi \geq 1$ are indispensable)

$$
\left(\sum_{i=1}^{N}\left\langle A_{i} R_{i}, u\right\rangle^{\chi}+\langle B, u\rangle^{\chi}\right)^{1 / \chi} \leq \sum_{i=1}^{N}\left\langle A_{i} R_{i}, u\right\rangle+\langle B, u\rangle .
$$

We need only to show that

$$
\int_{\mathbb{S}^{+}} \frac{1}{e_{*}^{\chi}(u)} \mathbb{E}\left(\langle B, u\rangle^{\chi}\right) \pi_{*}^{\chi}(d u)>0
$$

We will show that there exists $c_{\chi}>0$ such that

$$
\int_{\mathbb{S}^{+}}\langle x, u\rangle^{\chi} \pi_{*}^{\chi}(d u) \geq c_{\chi}\|x\|^{\chi},
$$

for every $x \in \mathbb{R}_{+}^{d}$. Observe that $\mathbb{S}^{+} \ni x \mapsto \int_{\mathbb{S}^{+}}\langle x, u\rangle^{\chi} \pi_{*}^{\chi}(d u)$ is continuous and nonzero for every $x \in \mathbb{S}^{+}$, since supp $\pi_{*}^{\chi}$ is not contained in any proper subspace of $\mathbb{S}^{+}$(see Sect. 2). This allows us to conclude that $x \mapsto \int_{\mathbb{S}^{+}}\langle x, u\rangle^{\chi} \pi_{*}^{\chi}(d u)$ attains its minimum $c_{\chi}>0$ on $\mathbb{S}^{+}$, and in fact this proves (5.23).

In order to prove (5.22) notice that by (5.23) we obtain

$$
\begin{aligned}
& \int_{\mathbb{S}^{+}} \frac{1}{e_{*}^{\chi}(u)} \mathbb{E}\left(\langle B, u\rangle^{\chi}\right) \pi_{*}^{\chi}(d u) \\
& \geq \frac{1}{\sup _{u \in \mathbb{S}^{+}} e_{*}^{\chi}(u)} \int_{\mathbb{S}^{+}} \mathbb{E}\left(\langle B, u\rangle^{\chi}\right) \pi_{*}^{\chi}(d u) \\
& \geq \frac{1}{\sup _{u \in \mathbb{S}^{+}} e_{*}^{\chi}(u)} \mathbb{E}\left(\int_{\mathbb{S}^{+}}\langle B, u\rangle^{\chi} \pi_{*}^{\chi}(d u)\right) \\
& \geq \frac{c_{\chi}}{\sup _{u \in \mathbb{S}^{+}} e_{*}^{\chi}(u)} \mathbb{E}\left(\|B\|^{\chi}\right)>0,
\end{aligned}
$$

since $\mathbb{P}(\{B>0\})>0$. This completes the proof of Theorem 1.9. 
Acknowledgments The results of this paper are part of the author's PhD thesis, written under the supervision of Ewa Damek at the University of Wroclaw. I wish to thank her for many stimulating conversations and several helpful suggestions during the preparation of this paper. I would like also to thank Dariusz Buraczewski for beneficial discussions and comments. The author is grateful to the referee for a very careful reading of the manuscript and useful remarks that lead to the improvement of the presentation.

Open Access This article is distributed under the terms of the Creative Commons Attribution License which permits any use, distribution, and reproduction in any medium, provided the original author(s) and the source are credited.

\section{References}

1. Bogachev, V.I.: Measure Theory. Springer, Berlin (2006)

2. Breiman, L.: The strong law of large numbers for a class of Markov chains. Ann. Math. Stat. 31(3), 801803 (1960)

3. Buraczewski, D., Damek, E., Guivarc'h, Y.: On multidimensional Mandelbrot's cascades. Preprint (2010). http://www.math.uni.wroc.pl/ dbura/publications/man110707.pdf

4. Buraczewski, D., Damek, E., Mentemeier, S., Mirek, M.: Heavy tailed solutions of multivariate smoothing transforms. Preprint (2012)

5. Goldie, Ch.M.: Implicit renewal theory and tails of solutions of random equations. Ann. Appl. Probab. 1(1), 126-166 (1991)

6. Guivarc'h, Y., Le Page, É.: Simplicité de spectres de Lyapounov et propriété d'isolation spectrale pour une famille d'opérateurs de transfert sur l'espace projectif. Random Walks and Geometry, pp. 181-259. Walter de Gruyter GmbH \& Co. KG, Berlin (2004)

7. Guivarc'h, Y., Le Page, É.: On matricial renewal theorems and tails of stationary measures for affine stochastic recursions. Preprint (2011)

8. Guivarc'h, Y., Raugi, A.: Products of Random Matrices: Convergence Theorems. Random Matrices and Their Applications (Brunswick, Maine, 1984). Contemporary Mathematics, vol. 50, pp. 31-54. American Mathematical Society, Providence (1986)

9. Guivarc'h, Y., Urban, R.: Semigroups actions on tori and stationary measures on projective spaces. Stud. Math. 171(1), 33-66 (2005)

10. Hennion, H.: Limit theorems for products of positive random matrices. Ann. Probab. 25(4), 15451587 (1997)

11. Hennion, H., Hervé, L.: Stable laws and products of positive random matrices. J. Theor. Probab. 21(4), 966-981 (2008)

12. Horn, R.A., Johnson, Ch.R.: Topics in Matrix Analysis. Cambridge University Press, London (1994)

13. Jelenković, P.R., Olvera-Cravioto, M.: Information ranking and power laws on trees. Adv. Appl. Probab. (2010, in press). http://arxiv.org/abs/0905.1738

14. Jelenković, P.R., Olvera-Cravioto, M.: Implicit renewal theorem and power tails on trees (2010). http:// arxiv.org/abs/1006.3295

15. Jelenković, P.R., Olvera-Cravioto, M.: Implicit renewal theorem for trees with general weights (2010). http://arxiv.org/abs/1012.2165

16. Kesten, H.: Random difference equations and renewal theory for products of random matrices. Acta Math. 131, 207-248 (1973)

17. Kesten, H.: Renewal theory for functionals of a Markov chain with general state space. Ann. Probab. 2, 355-386 (1974)

18. Liu, Q.: Asymptotic properties and absolute continuity of laws stable by random weighted mean. Stoch. Process. Appl. 95, 83-107 (2001) 